\title{
Foundational aspects of a multi-scale modeling framework for composite materials
}

\author{
Somnath Ghosh
}

\section{Correspondence:}

sghosh20@jhu.edu

Departments of Civil and

Mechanical Engineering, Johns

Hopkins University, Baltimore, MD

21218, USA

\section{说) Springer}

\begin{abstract}
The objective of this paper is to provide an integrated computational materials science and engineering or ICMSE perspective on various aspects governing multi-scale analysis of composite materials. These include microstructural characterization, micromechanical analysis of microstructural regions, and bridging length scales through hierarchical modeling. The paper discusses methods of identifying representative volume elements or RVEs in the material microstructure using both morphology- and micromechanics-based methods. For microstructures with nonuniform distributions, a statistically equivalent RVE or SERVE is identified for developing homogenized properties under undamaged and damaging conditions. A particularly novel development is the introduction of SERVE boundary conditions based on the statistical distribution of heterogeneities in the domain exterior to the SERVE. A micromechanical model of the SERVE incorporating explicit damage mechanisms like interfacial debonding, and fiber and matrix damage is developed for crack propagation. Finally, a microstructural homogenization-based continuum damage mechanics (HCDM) model is developed that accounts for the microstructural distributions as well as the evolution of damage. The HCDM model-based simulations are able to provide both macroscopic and microscopic information on evolving damage and failure.
\end{abstract}

Keywords: Statistically equivalent RVE; Statistical distribution-based SERVE boundary conditions; Cohesive zone models; Homogenization-based continuum damage mechanics (HCDM)

\section{Background}

Multi-scale modeling has become a familiar theme, integral to the modeling of heterogeneous materials, such as composites. The ability of powerful computational methods to resolve material behavior at different scales and communicate across them is fostering unprecedented advances in multi-scale modeling. These models provide in-depth understanding of material deformation and failure that can revolutionize integrated structure-material design. It is prudent to use the notion of multiple spatial scales in the analysis of composite materials and structures due to the inherent existence of various scales. Conventional methods of analysis have used effective properties obtained from homogenization of response at microscopic length scales. A number of analytical models have evolved within the framework of small deformation linear elasticity theory to predict homogenized macroscale constitutive response of heterogeneous materials, accounting for the characteristics of microstructural behavior. The underlying principle

(c) 2015 Ghosh. This is an Open Access article distributed under the terms of the Creative Commons Attribution License (http:// creativecommons.org/licenses/by/4.0), which permits unrestricted use, distribution, and reproduction in any medium, provided the original work is properly credited. 
of these models is the Hill-Mandel condition of homogeneity, which states that for large differences in microscopic and macroscopic length scales, the volume-averaged strain energy is obtained as the product of the volume-averaged stresses and strains in the representative volume element (RVE). Cogent reviews of various homogenization models are presented in [1]. Notable among the various estimates and bounds on the elastic properties are the variational approach using extremum principles [2,3], self-consistent model $[4,5]$, etc. These analytical models however do not provide adequate resolution to capture the fluctuations in microstructural variables that can have significant effects on properties.

The use of computational micromechanical methods like the finite element method, boundary element method, spring lattice models, etc. has become increasingly popular for accurate prediction of stresses, strains, and other evolving variables in composite materials. Within the framework of computational multi-spatial scale analyses, two categories of methods have emerged. The first group, known as 'hierarchical models', entails bottom-up coupling for transfer of information from lower to higher scales [6-12]. Homogenization theory is based on complete scale separation with implicit assumptions of uniformity of macroscopic variables. Uncoupling of governing equations at different scales is often achieved through incorporation of periodicity boundary conditions on the microscopic RVEs, implying periodic repetition of a local microstructural region. The models can simultaneously predict evolution of macroscale variables using homogenized material properties and microscale variables in the periodic microstructural RVE as a post-processor to the macroscopic analysis module. A subset of the hierarchical models has been branded as the ' $\mathrm{FE}^{2}$ multi-scale methods' in [13], where micromechanical RVE models are solved in every increment to obtain homogenized properties for macroscopic analysis. However, this method can be very expensive as it entails solving the RVE micromechanical problem for every element integration point in the computational domain. To overcome the limitations of prohibitive computational overhead, macroscopic constitutive laws of elastic damage and elastic-plastic damage have been developed in $[11,14]$ from homogenization of RVE response at microscopic scales. The constitutive models represent the effect of morphological features and evolving microstructural mechanisms through evolving, anisotropic homogenized parameters. These reducedorder constitutive models are significantly more efficient than the $\mathrm{FE}^{2}$-type models since they have limited information on microstructural morphology and do not have to solve the RVE problem in every step.

The second category of concurrent multi-scale modeling methods has been proposed for problems of heterogeneous materials involving high solution gradients in [15-22]. Concurrent multi-scale models differentiate between regions that require differential resolutions and invoke two-way (both bottom-up and top-down) coupling of scales. They introduce a platform for coherent, coupled analysis through substructuring of the computational domain into (a) regions of macroscopic analysis using homogenized material properties and (b) embedded local regions of detailed micromechanical modeling. Macroscopic analysis with homogenized constitutive models in regions of low deformation or stress gradients enhances the efficiency of the computational analysis due to reduced-order constitutive representation. Top-down localization, on the other hand, requires cascading down and embedding critical regions of localized damage or instability with explicit representation of the microstructure and micromechanisms. The 
computational model concurrently performs micromechanical analysis in these regions with direct interfaces to the surrounding homogenized region of macroscopic analysis [20-22]. In other approaches in [23,24], higher-order gradients have been introduced to regularize the material model. The present paper will focus on hierarchical models only and not discuss concurrent multi-scale models further.

Multi-scale modeling of composites, especially for structures and materials in extreme environments such as failure and fatigue loading conditions, requires detailed scalespecific models that incorporate the underpinnings of the microstructure on material behavior. A holistic approach requires both characterization and modeling at each relevant scale and consequently establishes bridges between them. Emergent thrusts in integrated computational materials science and engineering or ICMSE and virtual materials systems are fostering unprecedented advances, integrating microstructure representations, constitutive descriptions, computational algorithms, and experimental methods. The objective of this paper is to provide an ICMSE perspective on different aspects governing multi-scale analysis of composite materials. These include microstructural characterization, micromechanical analysis of microstructural regions, and bridging length scales involving bottom-up or hierarchical modeling. The paper begins with a discussion of different methods of identifying RVEs in the material microstructure using both morphology- and micromechanics-based methods. For microstructures with nonuniform distributions, a statistically equivalent RVE or SERVE is identified for developing homogenized properties under undamaged and damaging conditions. A particularly novel development is the introduction of SERVE boundary conditions based on the statistical distribution of heterogeneities in the domain exterior to the SERVE in the 'A novel boundary condition for defining SERVE based on statistical distribution of heterogeneities' section. Section 'Micromechanical model of SERVE undergoing damage and failure' develops a micromechanical model of the SERVE incorporating explicit damage mechanisms like interfacial debonding and fiber and matrix damage. Finally, a microstructural homogenization-based continuum damage model (HCDM) is developed in the 'Homogenization-based continuum damage mechanics model' section that accounts for the microstructural distributions as well as the evolution of damage. The HCDM model corresponds to diffused damage in the macrostructure and is not valid in regions of severe macroscopic localization. In such cases, a concurrent multi-scale model as developed by the authors in $[21,22]$ is desirable. The author has developed 3D HCDM model in [25-27] in a principal damage coordinate system (PDCS), which evolves with the load history. These are essential steps in developing rigorous multi-scale models of damage and failure in heterogeneous materials.

\section{Identification of the representative volume element or RVE for homogenization}

The microstructural RVE is an important characteristic in the determination of effective material properties [28-31]. The RVE depends on the material property of interest and can vary significantly from one class of properties to another, even for the same material microstructure. For example, the RVE for strength can be quite different from that for toughness. Identification of the appropriate RVE that locally represents the effect of the microstructure in an average sense is an essential part of homogenization. A large number of studies have been conducted with the RVE represented by a unit cell and consisting of a single heterogeneity in a regular (square, cubic, hexagonal, etc.) matrix . The 
underlying assumptions in these studies are that the microstructure depicts a uniform, periodically repetitive array of heterogeneities. In practice, however, the occurrence of perfect uniformity or periodicity is rare in heterogeneous microstructures. Even when geometric periodicity may exist, periodicity in the evolving variables, e.g. damage, may not hold. While it may be difficult or even impossible to identify the RVE for nonuniform microstructures shown in Figure 1a, it is important to identify statistically equivalent RVES or SERVEs to evaluate homogenized macroscopic properties. Methods of identifying statistically representative microstructural regions based on purely geometric and morphological considerations have been discussed in [32-34] using a combination of statistical and computational analyses.

A SERVE can be identified as the smallest volume element of the microstructure exhibiting the following characteristics.

1. Effective material properties, e.g., stress-strain behavior in the SERVE should be equivalent to the properties of the entire microstructure, at least locally.

2. Distribution functions of parameters reflecting the local morphology, like local volume fraction, neighbor distance, or radial distributions in the SERVE should be equivalent to those for the overall microstructure.

3. The SERVE should be independent of location in the local microstructure, as well as of the applied loading.

The second characteristic is driven by the postulate that response functions characterizing a material behavior should have a strong dependence on morphological parameters of the microstructure. Assuming that the SERVE corresponds to a converged property or response function, the set of distribution functions should depend on what set of morphological parameters, e.g., local/overall volume fraction, nearest neighbor distance, shape, etc., control that response function. The necessary set should be at least the parameters that most strongly affect that response function, while the sufficient set should consist of additional parameters that will have smaller effects on the behavior.

Figure 1 shows a $100 \mu \mathrm{m} \times 79.09 \mu \mathrm{m}$ optical micrograph of a steel fiber-reinforced epoxy matrix composite. The matrix material is an epoxy with Young's modulus: $E_{m}=4.6$ GPa and Poisson ratio: $v_{m}=0.4$. The steel fiber material has Young's modulus: $E_{f}=210$ GPa and Poisson ratio: $v_{f}=0.3$. All fibers are aligned perpendicular to the plane of

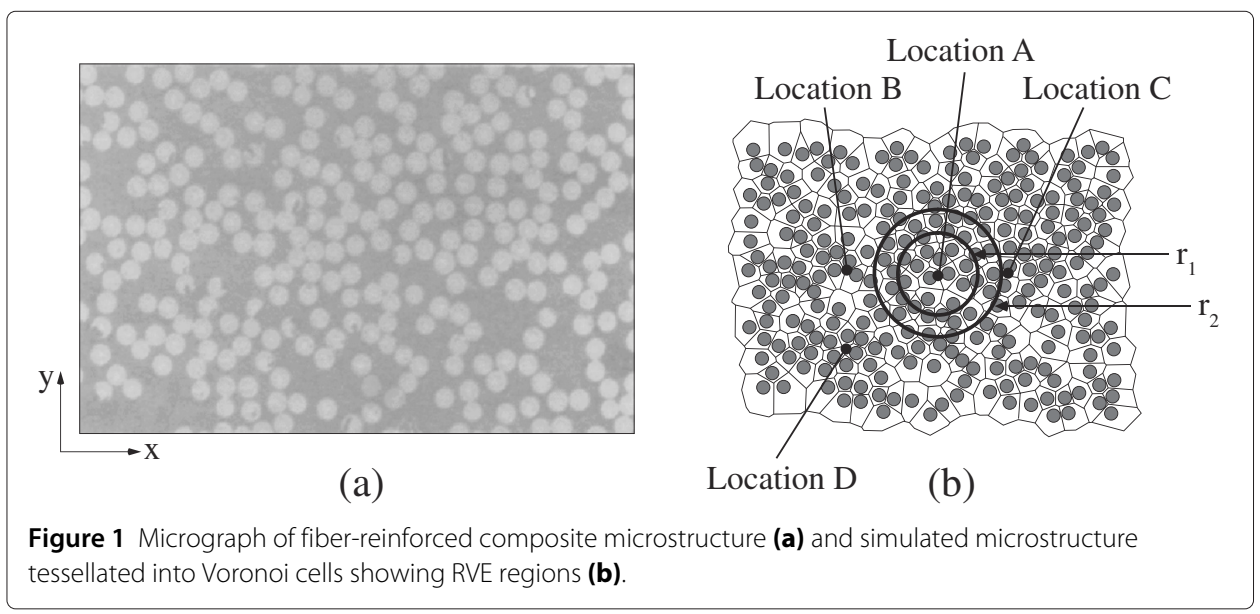




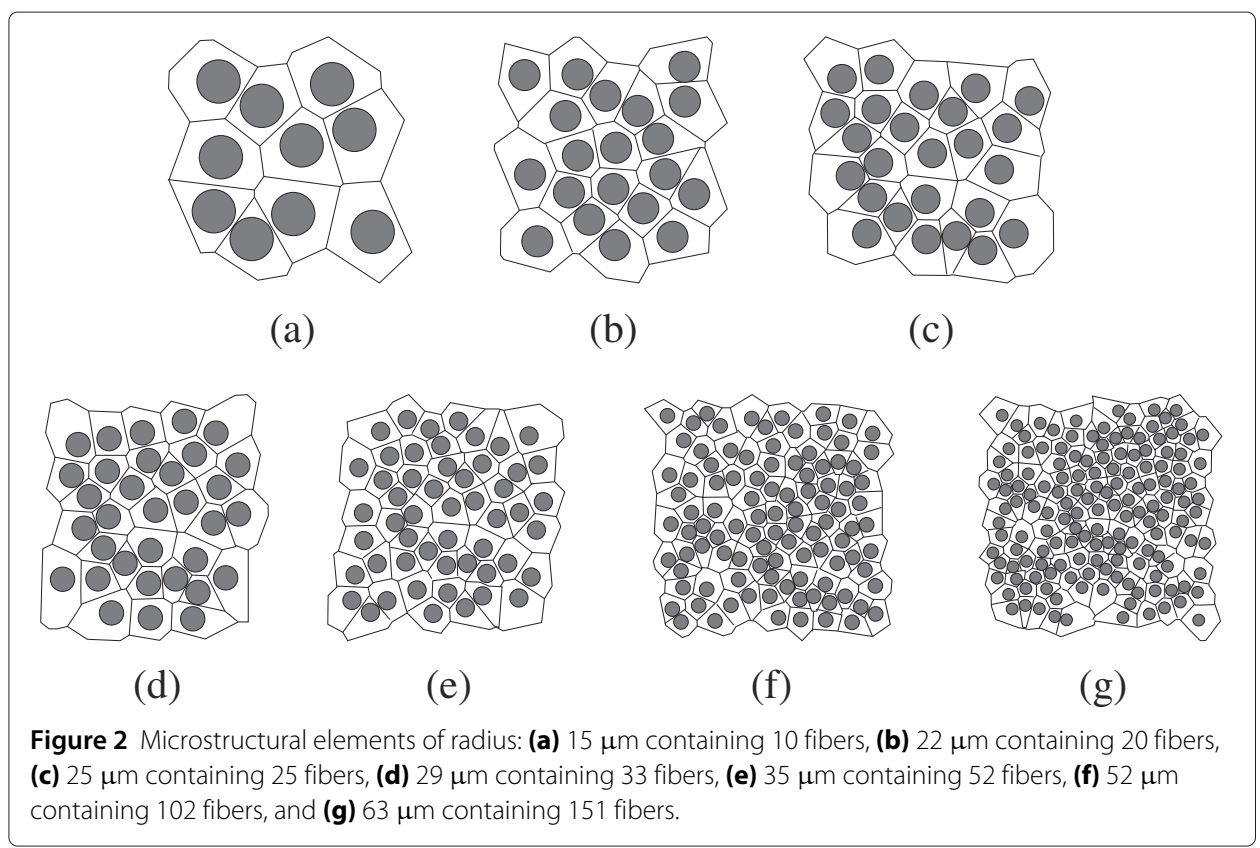

the paper and have circular cross sections with a radius of $1.75 \mu \mathrm{m}$. Figure $1 \mathrm{~b}$ shows a computer-generated image of the optical micrograph that is tessellated into a network of Voronoi cells [34]. The circular region is used to identify $N$ inclusions belonging to a SERVE. While satisfying different criteria may lead to nonuniqueness, it is possible to postulate the SERVE as the microregion that will satisfy all of the above requirements. Arriving at the optimal SERVE size is important to prevent risking erroneous estimation of effective properties with smaller RVEs or requiring huge computational resources with larger RVEs. Two metrics are discussed here for identification of the SERVE in undamaged and damaged composite microstructures.

\section{Convergence of homogenized tangent stiffness tensor}

Domains for which the effective homogenized stiffness tensor $\left[E_{i j k l}^{H}\right]$ converges to that for the entire microstructure, at least locally, is an important metric in SERVE estimation. For a given heterogeneous microstructure, the stiffness tensor $\left[E_{i j k l}^{H}\right.$ ] may be evaluated by volume averaging the microscopic stresses $\left[\hat{\sigma}_{i j}\right]$ generated by applying periodic boundary conditions corresponding to a unit macroscopic strain. Figure 2a,b,c,d,e,f,g illustrates windows of different sizes that are extracted from the microstructure of Figure 1a corresponding to the number $N$ of inclusions in the SERVE. These are created from concentric circles of radius $r$ in Figure 2b. The smallest window size of approximately $15 \mu \mathrm{m}$ contains 10 fibers, while the largest $63-\mu \mathrm{m}$ window contains 150 fibers. Table 1 compares the area fractions of different windows with that of the micrograph.

Table 1 Size (in $\mu \mathrm{m}$ ), number of fibers, and area fraction of RVEs

\begin{tabular}{ccccccccc}
\hline Size & $\mathbf{1 5}$ & $\mathbf{2 2}$ & $\mathbf{2 5}$ & $\mathbf{2 9}$ & $\mathbf{3 5}$ & $\mathbf{5 2}$ & $\mathbf{6 3}$ & Micrograph \\
\hline \# fibers & 10 & 20 & 25 & 35 & 50 & 100 & 150 & 264 \\
AF (\%) & 31.5 & 32.1 & 32.2 & 31.5 & 33.1 & 32.3 & 31.3 & 32.3 \\
\hline
\end{tabular}


A special method of constructing a periodic computational domain with nonstraight edges, containing periodically positioned nodes for the nonuniform microstructure, has been developed in $[19,34,35]$. Each of the $N$ inclusions in the core region is repeated in the $x$ and $y$ directions for a few period lengths. The extended domain of multiply repeated SERVEs is tessellated into a network of Voronoi cells. The boundary of the central SERVE is generated as the aggregate of the outside edges of all the Voronoi cells associated with inclusions near the boundary as shown in Figure 2. The corresponding nodes on the boundary are periodic. The boundary of the domain is subjected to periodic conditions by constraining points on the boundary to displace periodically. The phase volume fraction in each of the windows is chosen to match the volume fraction of the overall microstructure to within a prescribed tolerance as tallied in Table 1. For each window, components of the effective stiffness tensor are evaluated and convergence is established through a comparison of the Frobenius norm of the homogenized stiffness tensor, defined as:

$$
\left\|E^{H}\right\|=\sqrt{\sum_{i=1}^{3} \sum_{j=1}^{3} \sum_{k=1}^{3} \sum_{l=1}^{3}\left(E_{i j k l}^{H}\right)^{2}}
$$

The SERVE is identified as the smallest window for which the difference in the norm, satisfy the criteria:

$$
\frac{\left\|E^{H}\right\|^{\text {micrograph }}-\left\|E^{H}\right\|^{\mathrm{RVE}}}{\left\|E^{H}\right\|^{\text {micrograph }}} \leq \mathrm{TOL}
$$

The superscript micrograph corresponds to the entire local micrograph. A tolerance of TOL $=5 \%$ is taken in this study. The SERVE is constructed for a number of locations randomly chosen in the micrograph. The window that satisfies the required characteristics is considered as the SERVE for the local microstructure. For undamaged microstructures, convergence of the Frobenius norm $\left\|E^{H}\right\|$ in Equation (2) and the corresponding deviations at three different locations are shown in Figure 3a,b. The windows containing 52 or more fibers exhibit less than $2 \%$ deviation from the stiffness of the entire micrograph. A region of radius $35 \mu \mathrm{m}$, encompassing 52 fibers, is found to exhibit converged stiffness components and be location independent.

Estimation of the SERVE for microstructures with evolving damage is a more extensive exercise $[33,34]$. Geometric parameters play a lesser role since the evolution of stresses and strains is affected by the distribution of evolving damage as well. Even when the
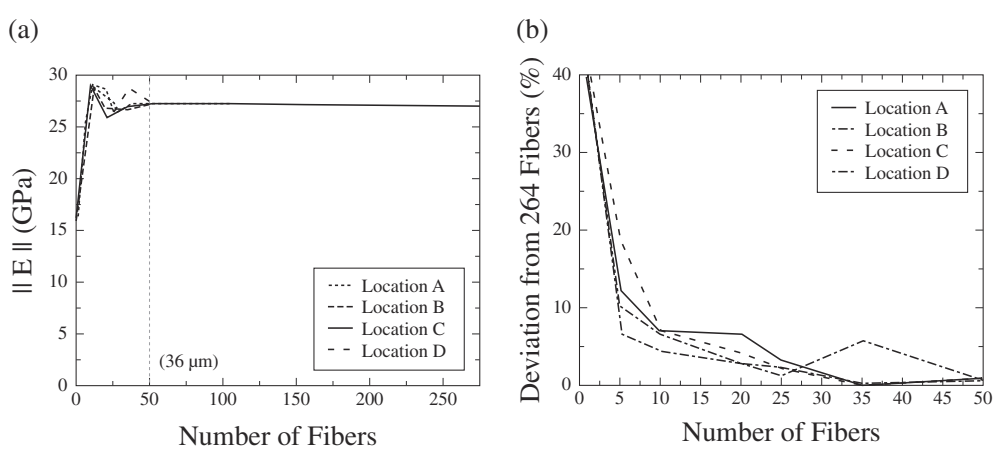

Figure 3 Plots showing (a) convergence of $\left\|E^{H}\right\|$ with increasing size, (b) deviation of local $\left\|E^{H}\right\|$ from the micrograph. 
microstructure is geometrically uniform, initiation and progression of damage can result in a SERVE that is considerably larger than a unit cell. Analysis of the SERVE for heterogeneous microstructures undergoing interfacial debonding has been discussed in $[33,34]$ using approaches similar to those used for the undamaged material. Initiation and progression of damage in the microstructure require the consideration of an evolving SERVE. Convergence of the degrading homogenized stiffness tensor $\left[E_{i j k l}^{H}\right]$ is taken as an indicator of the region of influence and hence is a metric for estimating the SERVE. Instead of the tangent stiffness tensor, $\left[E_{i j k l}^{H}\right]$ is represented as the linear unloading stiffness tensor from the point of loading in the macroscopic stress-strain plot.

Periodic windows of increasing sizes ranging from approximately $22 \mu \mathrm{m}$ containing 20 fibers to $63 \mu \mathrm{m}$ containing 150 fibers are analyzed for three different strain histories, viz. increasing $\epsilon_{x x}, \epsilon_{y y}$, and $\epsilon_{x y}$, respectively. The stiffness tensor in unloading is evaluated at the terminal values of the macroscopic strain for the entire microstructure as well as for each increasing window. The SERVE size changes with increasing damage induced strain as shown in Figure 4. At low strains, the SERVE is of size $35 \mu \mathrm{m}$, containing about 52 fibers for all the three strain conditions. However, the SERVE size increases at a much slower rate for the shear strain condition than for the normal strains. This is a consequence of the extent of damage with these strain conditions, which in turn is also a function of the cohesive law parameters for the interface. The SERVE size depends on the material state that is governed by damage. For a given strain value, the SERVE size corresponds to that load path for which the damage state is maximum. It may be able to represent the SERVE size as a function of the evolving damage, which defines material state. The SERVE size will converge when damage is contained.

\section{Convergence of statistical functions of microstructural variables}

The marked correlation function $M(r)$ has been introduced in [36,37] for providing multi-variate characterization of the microstructural phase distribution. These functions correlate any chosen field variable like stress, strain, or a function dependent on the morphology of the microstructure. Specifically, they characterize the region of influence in a heterogeneous neighborhood with respect to fields of evolving variables. The marked correlation function $M(r)$ is expressed as the ratio of state variable and geometric distribution functions as:

$$
M(r)=\frac{h(r)}{g(r)}
$$

(a)

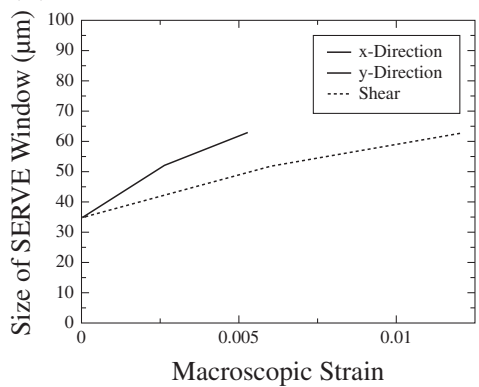

(b)

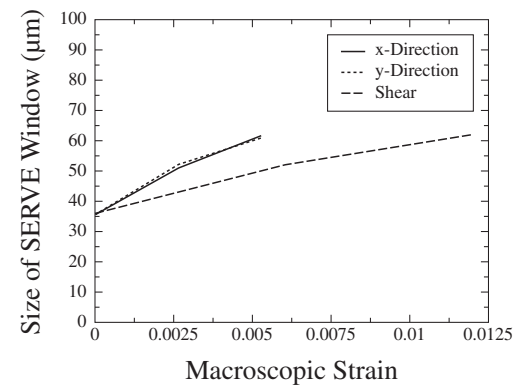

Figure 4 SERVE size for normal and shear macroscopic strains from (a) convergence of unloading stiffness, (b) $M(r)$. 
where the state variable dependent function $h(r)$ is derived from the mark intensity function $H(r)$ as:

$$
h(r)=\frac{1}{2 \pi r} \frac{d H(r)}{d r} \text { and } H(r)=\frac{1}{m^{2}} \frac{A}{N^{2}} \sum_{i}^{N} \sum_{j=1}^{j_{i}} m_{i} m_{j}(r)
$$

Here, $m_{i}$ represents a 'mark' associated with the $i$-th inclusion. A mark can be any chosen state variable field that is relevant to the specific properties in question. For observations within a finite window of area $A$, the variable $r$ is a measure of the radial distance of influence and $m_{j}(r)$ corresponds to the mark associated with the $j$-th inclusion at a radial distance $r$. In Equation (4), $m$ is the mean of all marks, $N$ is the total number of inclusions, and $j_{i}$ is the number of inclusions that have their center within a circle of radius $r$ centered at the $i$-th inclusion. The pair distribution function $g(r)$ corresponds to the probability $g(r) d r$ of finding an additional inclusion center between concentric ring of radii $r$ and $r+d r$, respectively. It characterizes the occurrence intensity of inter-inclusion distances and is expressed as:

$$
g(r)=\frac{1}{2 \pi r} \frac{d K(r)}{d r} \quad \text { where } \quad K(r)=\frac{A}{N^{2}} \sum_{k=1}^{N} I_{k}(r)
$$

$I_{k}(r)$ is the number of additional inclusion centers that lie inside a circle of radius $r$ about an arbitrarily chosen inclusion. $K(r)$ is a second-order intensity function, defined in [36] as the number of additional inclusions that lie within a distance $r$ of an inclusion center and divided by the number density $N / A$ of inclusions.

A declining value of $M(r)$ indicates reduced correlation between elements of the microstructure. It is therefore a good metric for the estimation of SERVE or the region of influence for a nonuniform microstructure. Steps to evaluate the SERVE size from $M(r)$ have been described in [32,34]. For a random distribution, the pair distribution function $g(r)$ approaches unity at large radial distances $r$. The radius of convergence $r_{0}$ is identified from the $g(r)$ plot for which $g(r) \approx 1$ for $r>r_{0}$. Appropriate microstructural variable fields associated with each inclusion are assigned as a 'mark', e.g., principal stresses and strains, Von Mises stress, etc. $M(r)=1$ corresponds to an uncorrelated random distribution of circular heterogeneities having identical marks. For nonuniform microstructures, $M(r)$ stabilizes to near-unit values at a radius of convergence $r_{p}$, such that for $r>r_{p}$, $M(r) \approx 1$ and the local morphology ceases to have significant influence on the state variables. The radius $r_{p}$ corresponds to the length-scale of correlation between the physical behavior and microstructural morphology. It provides an estimate for SERVE size.

\section{Marked correlation function with geometric parameter-based marks}

While stress or traction-based marks yield satisfactory estimates of the SERVE, it is of interest to see if similar results can be generated by using marks that are purely geometric parameters. This conjecture is based on the premise that the response function should be governed by morphological aspects of the microstructure. If a local response variable depends on a certain local morphological characteristic or a combination thereof, then similar outcomes for physical and geometric marks may indicate that full blown analyses are not needed to estimate the SERVE. A weighted function of geometric parameters is 
constructed as a mark in $[32,38]$ to represent geometric parameters which contribute to the initiation of damage. The mark associated with the $k$-th inclusion is defined as:

$$
m_{k}=w_{1} S_{1}^{k}+w_{2} S_{2}^{k}+w_{3} S_{3}^{k}
$$

where $S_{i}^{k}$ are geometric parameters characterizing the local distribution and $w_{i}$ are associated weights. In Equation (6), $S_{1}^{k}$ is a measure of the normalized local area fraction for the $k$-th inclusion defined as:

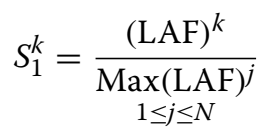

$N$ is the total number of inclusions, and (LAF) ${ }^{j}$ is the local area fraction for the $j$-th inclusion. The area fraction is evaluated as the ratio of the inclusion cross-sectional area to the area of the associated Voronoi cell. $S_{2}^{k}$ is a normalized measure of the inverse of near-neighbor distance for the $k$-th inclusion, i.e.

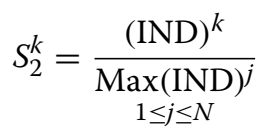

where (IND) $)^{j}$ is the inverse of the near-neighbor distance of the $j$-th inclusion. Nearneighbors of an inclusion are those that share common edges of the Voronoi cell. The near-neighbor distance is the average of the distances between an inclusion and its neighbors. $S_{3}^{k}$ is a normalized measure of the number of near-neighbors for the $k$-th inclusion, given as:

$$
S_{3}^{k}=\frac{(\mathrm{NN})^{k}}{\underset{1 \leq j \leq N}{\operatorname{Max}(\mathrm{NN})^{j}}}
$$

where $(\mathrm{NN})^{j}$ is number of near-neighbors for the $j$-th inclusion. The number of nearneighbors is the number of Voronoi cell edges. Figure 5 compares $g(r)$ for a Poisson distribution of points $(g(r)=1)$ with those for the micrograph where the edge effect is accounted for. At lower values of $r$, there is a significant deviation of the plots from unit value due to short-range geometric disorders such as clustering. Convergence is assumed to occur if the percentage change in incremental area under each $g(r)$ or $M(r)$ curve is

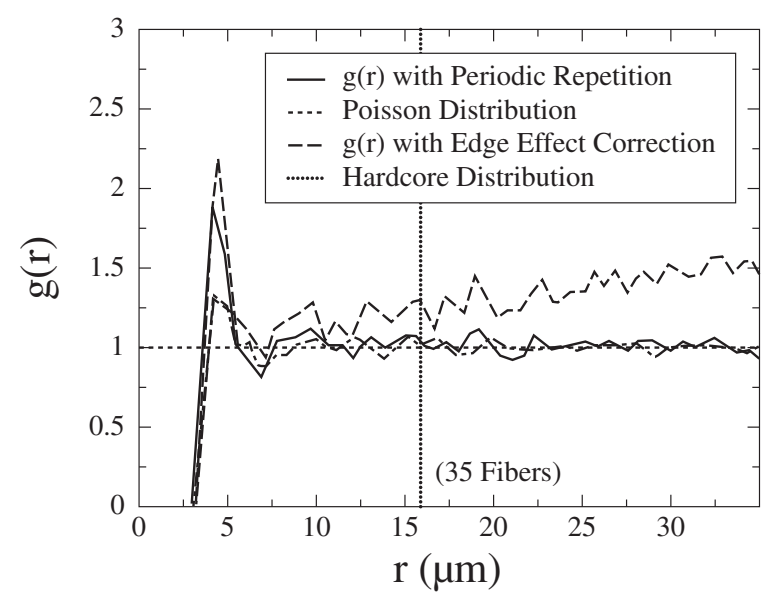

Figure 5 Plots of $g(r)$ with two methods of edge correction compared with Poisson's distribution. 
below a tolerance of 5\%. The function $g(r)$ converges to unity with increasing values of $r$ $(>15 \mu \mathrm{m})$ containing about 35 fibers.

The maximum traction at the fiber-matrix interface is a good indicator of interfacial debonding initiation. Hence, this is considered as a candidate mark in $M(r)$. Traction is evaluated as the resultant of the normal tensile and tangential components, i.e., $\sqrt{T_{n}^{2}+T_{t}^{2}}$. The mark is taken as the average traction at a set of points on the fiber-matrix interface that experiences the highest tensile normal components. Figure 6 shows a plot of $M(r)$ vs. $r$ for different fractions of points on the interface under different macroscopic strain conditions. The line $M(r)=1$ corresponds to a uniform distribution of fibers with identical marks. In Figure 6a, $M(r)$ is constructed by averaging the tractions respectively at $1 \%$ and $10 \%$ of all points at the fiber-matrix interface that experience maximum traction. The macroscopic strain field is $\varepsilon_{x x}=1$, with all other components equal to zero. The two marks result in very similar $M(r)$ distribution, and both converge to unity approximately at a radius of convergence $r_{p} \approx 18 \mu \mathrm{m}$, containing about 50 fibers. Figure $6 \mathrm{~b}$ compares the distribution of $M(r)$ for three different applied uniaxial macroscopic strains. The mark in this case is the average traction at $3 \%$ of all points on the interface. For low values of $r$, the $M(r)$ plots for the three strains do not have the same distribution. This is attributed to the directional dependency of the mark with loading directions. With increasing $r$, the local anisotropy in the mark diminishes and all three plots converge to depict the same trend for $M(r)$. The radius of convergence for all the cases are approximately $r_{p} \approx 18 \mu \mathrm{m}$, containing about 50 fibers.

In summary, similar sizes of the SERVE are predicted by these alternate methods for given response functions. The successful use of the geometry-based indicators for problems without significant microstructural evolution, point to the fact that the morphological parameters strongly affect the response functions considered in the estimation of the SERVE. In these cases, the SERVE can be estimated without having to solve the entire micromechanics problem multiple times.

\section{Marked correlation function with damage variables}

$M(r)$ may also be used for estimating the SERVE in the presence of evolving damage, for which the 'mark' is chosen as a characteristic damage variable that evolves with increasing strain. Damage is considered for interfacial debonding only for this evaluation. The mark assigned is the inverse of the magnitude of the tensile traction that is averaged over

(a)

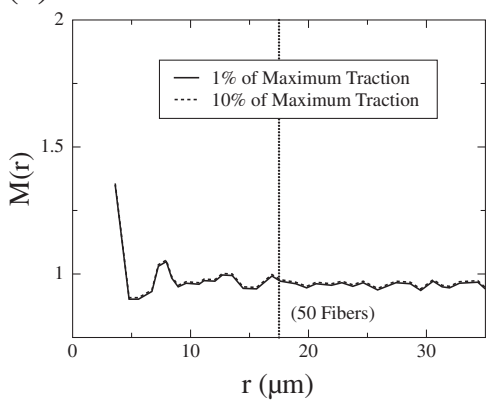

(b)

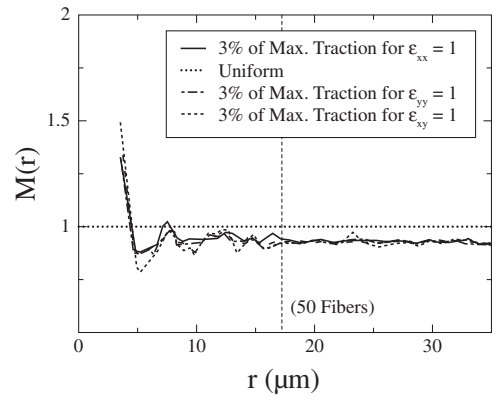

Figure $6 M(r)$ for (a) average traction of $1 \%$ and 10\% of maximum traction and (b) applied unit normal and shear strains. 
the length of the nondebonded or intact portion of interface. Only points that have tensile normal components at a inclusion-matrix interface are considered for the traction calculation. These traction values may lie either in the hardening or softening regions of the interfacial cohesive zone model. In the softening region, the tractions will decrease with increasing displacement jump and eventually reduce to zero, signaling the onset of microstructural damage due to interfacial debonding. With the evolution of debonding, tractions at the intact portion in the interface tend to lie in the softening region of the cohesive zone model and will have values that are close to zero. Consequently, the average traction in the intact portion of the interface will give a quantitative measure of the closeness to debonding and hence is represented by this mark. The marked correlation function $M(r)$ is evaluated for the micrograph at different strain intervals, with mark chosen as the inverse of the averaged traction over intact interfaces. Plots of $M(r)$ for the terminal values of applied macroscopic normal and shear strains are depicted in Figure 7. Convergence of $M(r)$ is assumed if the percentage increase of incremental area under each $M(r)$ curve is below $5 \%$ tolerance. All three $M(r)$ plots converge to unity $(M(r)=1)$ at a radius of convergence $r_{p}$ of approximately $31 \mu \mathrm{m}$, containing about 145 fibers. The radius of convergence $r_{p}$ monotonically increases with damage evolution in the microstructure as shown in Figure $4 \mathrm{~b}$. The SERVE size at the terminal strain is approximately $62 \mu \mathrm{m}$, which is almost 1.7 times higher that for the undamaged interface approximately $36 \mu \mathrm{m}$. The rate of increase in SERVE size is found to be similar for the applied normal strains but is slower for the shear strain. Also, Figure 7 shows that the values of $M(r)$ for the three loading cases significantly differ until the convergence radius $r_{p}$ is reached. This is attributed to the dependence of mark values on the direction of applied loading below the radius $r_{p}$.

\section{A novel boundary condition for defining SERVE based on statistical distribution of heterogeneities}

Methods for determination of the SERVE in the previous section focused on the evaluation of the volume and material content in the microstructure. No consideration is given to the appropriateness of the boundary conditions applied to the SERVE for determining

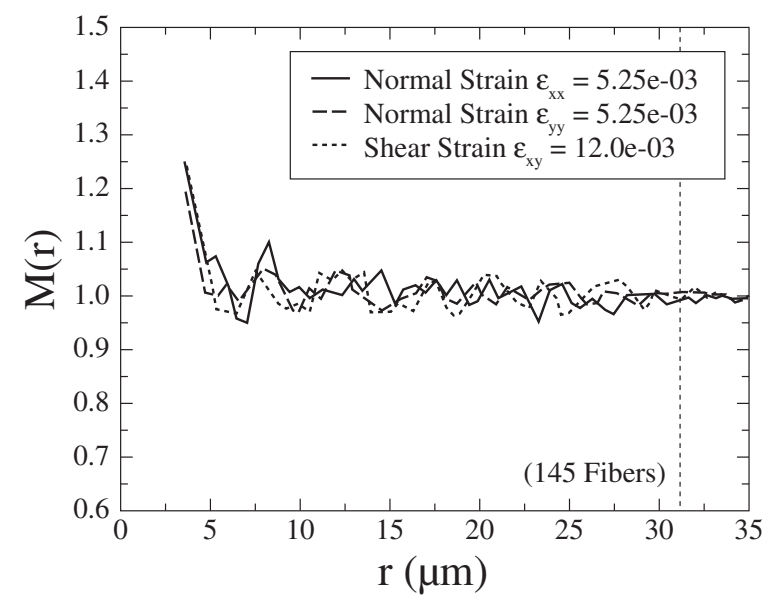

Figure $7 M(r)$ for applied normal and shear strains for inverse of averaged traction over intact surface. 
equivalent properties. Conventionally, three types of boundary conditions are applied on the SERVE. These are:

1. Affine displacement boundary conditions, $u_{i}^{0}=\epsilon_{i j}^{0} x_{j}$ on $\partial \Omega$, where $\epsilon_{i j}^{0}$ is a constant applied far-field strain and $x_{j}$ are the boundary positions, measured from the geometrical centroid of the RVE.

2. Uniform traction boundary condition $T_{i}=\sigma_{i j}^{0} n_{j}$ on $\partial \Omega$, where $\sigma_{i j}^{0}$ is the constant applied stress, $n_{j}$ is the unit normal to the boundary of the RVE, and $T_{i}$ is the applied traction on the RVE boundary.

3. Periodic boundary conditions $u_{i}^{p}=\epsilon_{i j}^{0} x_{j}+u_{i}^{p d}$ on $\partial \Omega$, with a periodic additional displacement $u_{i}^{p d}$ which are equal on opposite faces of the RVE.

Typically, the uniform traction case gives the upper bound (Reuss bound), and the uniform strain condition provides the lower bound (Voigt bounds). These are not accurate for heterogeneous materials with nonuniform distributions. A drawback of the periodic boundary condition is that it automatically repeats the microstructure and associated deformation and damage patterns in the domain exterior to the RVE. This may not be accurate for nonuniform microstructures in general. Application of the above boundary conditions can result in an over-estimation of the RVE region due to convergence requirements. Accurate boundary conditions play an important role in determining the SERVE size. In an attempt to redefine the SERVE with respect to both the volume and boundary conditions, this section introduces a novel boundary condition required to meet the criteria described in the 'Identification of the representative volume element or RVE for homogenization' section. Optimally, the SERVE should (a) encompass the region required to represent essential deformation mechanisms and (b) represent boundary conditions that reflect the effect of the region exterior to the SERVE domain.

Various statistical descriptors such as distributions of the volume or area-fraction, nearest-neighbor-distance, etc. can be used to characterize the microstructural morphology of nonuniformly dispersed composites. In $[39,40]$, it has been discussed that the spatial statistics of a two-phase random medium can be completely described by specifying only the volume fraction and standard two-point correlation function $S_{2}(r)$. For multi-phase microstructures, the two-point correlation function is defined as the probability that two points at positions $\mathbf{x}_{1}$ and $\mathbf{x}_{2}$ and separated by a distance $r=\left|\mathbf{x}_{1}-\mathbf{x}_{2}\right|$ are found in the phase $i$ for a given orientation. In general, this function is able to characterize anisotropy due to its dependence on the orientation as opposed to the radial distribution function that is considered for isotropic distributions. In this study, it is assumed that the distribution of heterogeneities is statistically homogeneous and isotropic due to randomness of distribution. Hence, it is computed by repeatedly placing line segments of length $r$ randomly on the image of the microstructure. The two-point correlation for a statistically homogeneous and isotropic medium is mathematically expressed as:

$$
S_{2}(r)=\int_{\Omega} \iota^{F_{I}}(r) \iota^{F_{J}}\left(r+y_{J}\right) d \Omega
$$

where $\Omega$ is the total volume of the domain, $\iota^{F_{I}}=1 \forall x \in F_{I}$ is a phase indicator function, which is equal to unity inside the fiber $I$ and zero everywhere else in the domain. The two-point correlation function for the microstructure of Figure 8a is plotted in Figure 8b. 
(a)

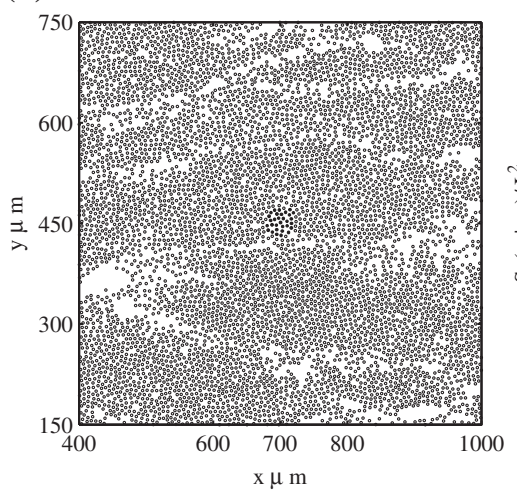

(b)

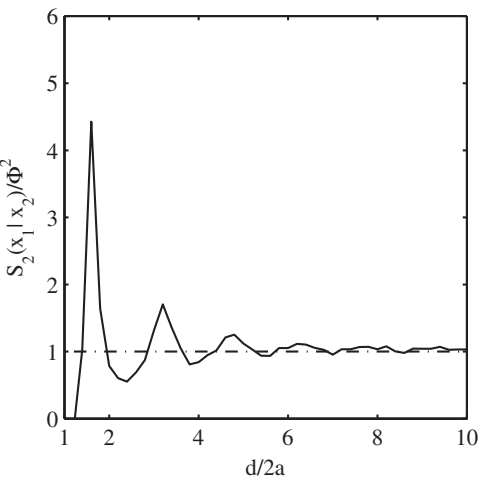

Figure 8 Microstructure of a nonuniformly dispersed unidirectional fiber-reinforced composite and two-point correlation of the microstructure. (a) Microstructure of a nonuniformly dispersed unidirectional fiber-reinforced composite, with 21,000 fibers of radius $2 \mu \mathrm{m}$. Two regions are depicted, viz. the SERVE domain containing 31 fibers and an exterior domain; (b) two-point correlation of the microstructure for the entire domain.

Displacement boundary conditions are imposed on the SERVE as an augmentation of the affine displacements through the expression $u_{i}=u_{i}^{0}+u_{i}^{*}$. Here, the augmented displacement field $u_{i}^{*}$ is derived by accounting for the microstructural distribution of the domain exterior to the RVE through a Green's function-based approach. While this approach of describing the SERVE boundary conditions is applicable to general threedimensional problems with inclusions of arbitrary shapes, the present study is restricted to unidirectional cylindrical fibers. For the sake of simplicity, all the fibers are assumed to have identical elastic properties, specifically stiffness components $C_{i j k l}^{F}$ and same crosssectional radius $a$. The fibers are however dispersed randomly in the matrix as seen from the microstructure in Figure 8a.

The pair-wise interaction of fibers 1 and 2, whose centroidal separation distance is $d_{12}$ is shown in Figure 9. The goal is to obtain the augmented displacement field due to the interacting pair of fibers at an observation point in the matrix denoted by $o$ in Figure 9 . The separation distance $d_{12}$ is random and may be characterized by statistical descriptors. The distance between the second fiber and the observation point $d_{2 o}$ (obtained from the law of triangles) is also random as $d_{12}$ is random. The Green's function solution to the interaction of the fibers can be obtained in terms of the eigen-strains $\epsilon_{k l}^{\Lambda}$ in the fibers, which are related to the far-field applied strain $\epsilon_{k l}^{0}$ as:

$$
\int_{\Omega}\left(M_{i j k l}+H_{i j k l}\right) \epsilon_{k l}^{\Lambda_{F_{I}} l_{I}}(x) d \Omega=-\epsilon_{i j}^{0}
$$

where the tensor $M_{i j k l}=\left(C_{i j p q}^{F}{ }^{l_{I}}(x)-C_{i j p q}^{M}\right)^{-1} C_{p q k l}^{M}$ depends on the fiber and matrix stiffness tensors $C_{i j k l}^{F}$ and $C_{i j k l}^{M}$, respectively, and

$$
H_{i j k l}=S_{i j k l} l^{F_{I}}(x)+G_{i j k l}\left(1-\iota^{F_{J}}(x)\right)
$$

This tensor is obtained from the Green's function solution of the interacting fiber-pair in Figure 9. The tensors $S_{i j k l}$ and $G_{i j k l}$ correspond to the interior- and exterior-Eshelby tensors, respectively. The distance of separation between the fibers ( 1 and 2) $d_{12}$ is random 


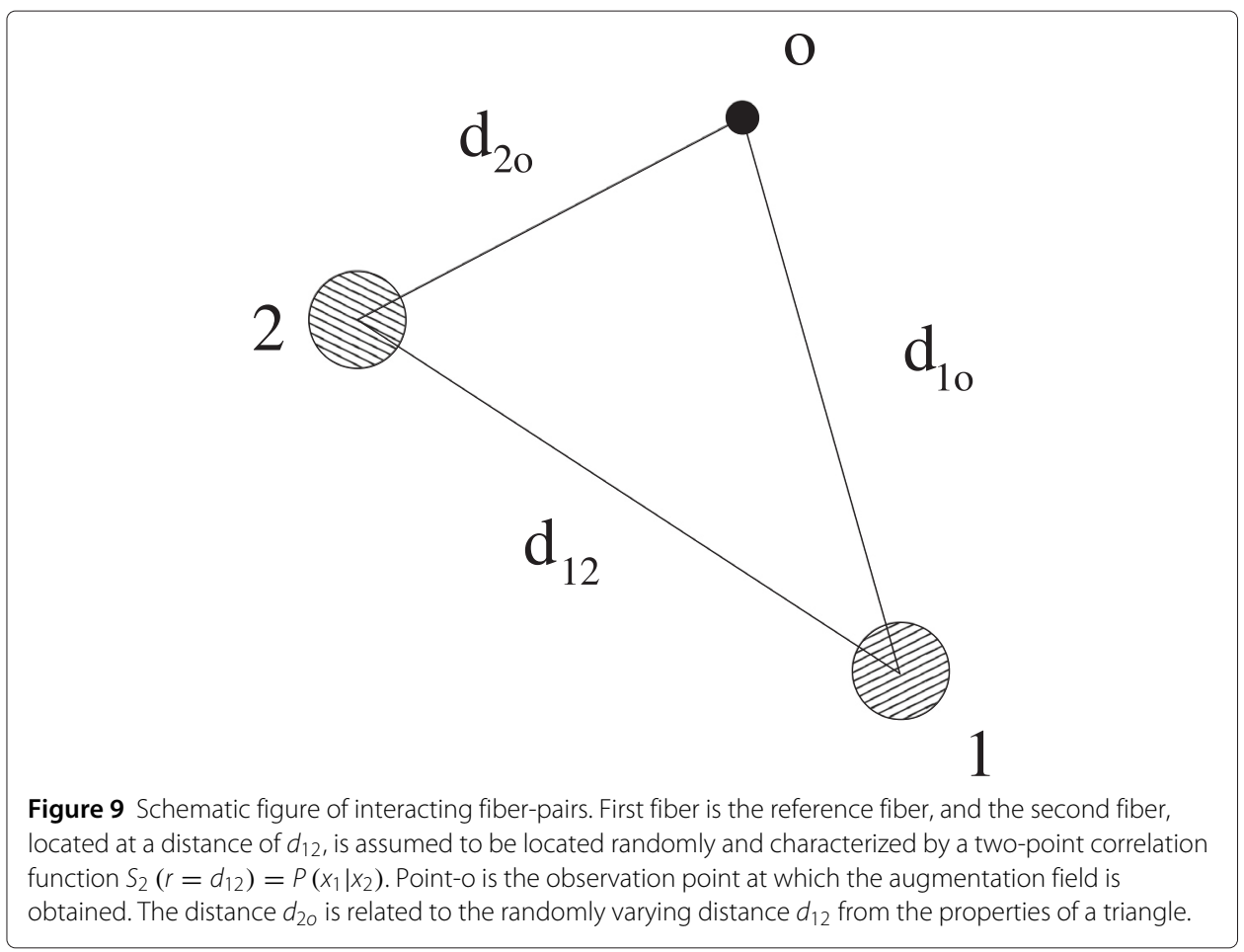

and characterized by the two-point correlation function. The augmented strain $\epsilon_{i j}^{*}$ in terms of the two-point correlation function is given as:

$$
\epsilon_{i j}^{*}(x)=\int_{\Omega \backslash \Omega_{1}}\left[\int_{\Omega}\left(M_{i j p q}+H_{i j p q}\right) d \Omega\right]^{-1} S_{p q k l} S_{2}\left(x \mid x^{\prime}\right) \epsilon_{k l}^{0} d x^{\prime}
$$

The corresponding displacement field $u_{i}^{*}$ that augments the affine displacements is expressed as:

$$
u_{i}^{*}(x)=\int_{\Omega \backslash \Omega_{1}}\left[\int_{\Omega}\left(M_{i j p q}+H_{i j p q}\right) d \Omega\right]^{-1} L_{p q l} S_{2}\left(x \mid x^{\prime}\right) \epsilon_{l j}^{0} d x^{\prime}
$$

In Equation (14), the third-order tensor $L_{p q l}$ relates the disturbance displacement to the eigen-strains $\epsilon_{k l}^{\Lambda}$. The tensor $L_{p q l}$ is obtained from the Green's function solution of the interacting fibers.

To illustrate the results of the augmented SERVE boundary conditions, a systematic convergence study is conducted by increasing the number of fibers $N_{f}$ and corresponding SERVE size $S$. The ratio of the fiber and matrix Young's moduli for this problem is set to be $E^{F} / E^{M}=25$, and the Poisson's ratio of the fiber and matrix is $v^{F}=0.25$ and $v^{M}=0.4$, respectively. Parametric finite element simulations are performed using the ABAQUS-Standard commercial code. The size of the RVE is systematically increased from $S=35 \mu \mathrm{m}\left(N_{f}=17\right)$ to $S=165 \mu \mathrm{m}\left(N_{f}=499\right)$. The number of fibers $N_{f}$ and the size of the RVE $S$ are related to each other through the fiber volume fraction $\phi$ and fiber radius $a$ since $S^{2}=\pi N_{f} a^{2} / \phi$. The SERVEs are subjected to both the affine- and the statistical distribution augmented displacement boundary conditions, respectively. The results of the convergence study are illustrated in Figure 10. The figure plots the normalized homogenized stiffness component as a function of the SERVE size $S$ and number of fibers $N_{f}$. The homogenized moduli are normalized with those of the pure matrix $\left(N_{f}=0\right)$. 


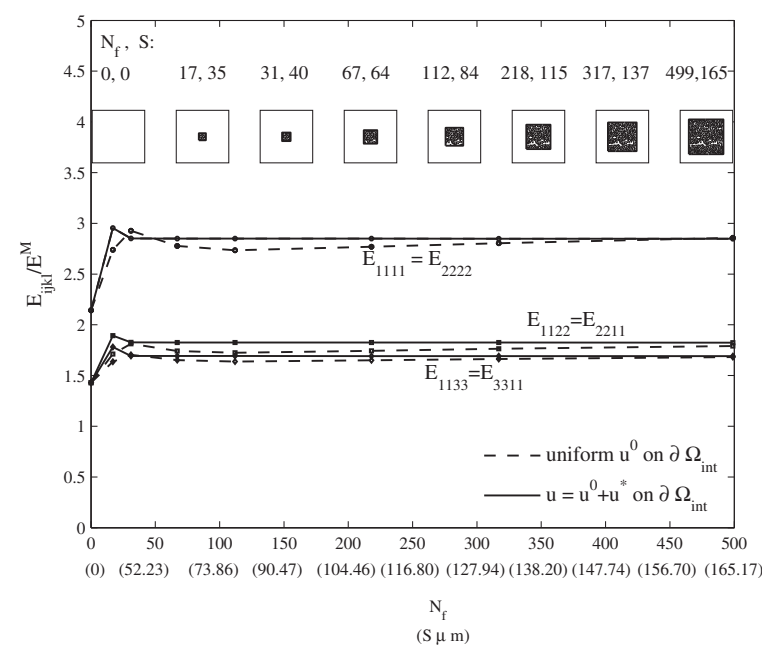

Figure 10 Normalized homogenized stiffness components as functions of SERVE size $S$ and number of fibers $N_{f}$.

The homogenized modulus converges to a fixed value for $S \geq 84 \mu \mathrm{m}$ with $N_{f} \geq 112$ for the affine displacement as shown in dashed lines of the figure. However, with the statistical Green's function-based-augmented displacement boundary conditions, the SERVE size converges at $S=40 \mu \mathrm{m}$ with $N_{f}=31$. This elucidates the strong effect of the realistic boundary conditions on the SERVE. This could potentially be a new way of defining a SERVE and will be tested in future work.

\section{Micromechanical model of SERVE undergoing damage and failure}

Micromechanical analyses of the identified composite microstructural SERVE are necessary ingredients for the development of a homogenization-based continuum damage mechanics or HCDM model. This section briefly discusses the computational models that describe the deformation and damage response of fiber-reinforced matrix composites exhibiting fiber and matrix cracking as well as interfacial debonding.

\section{Constitutive model for fiber and matrix in the composite microstructure}

The mechanical behavior of the fiber and matrix is characterized by high stiffness and strength and low failure strain. The failure process is short, and the stress drops abruptly after the inception of brittle fracture. The brittle fiber and matrix are assumed to be isotropic, elastic undergoing small deformation. The stress-strain relation is given as:

$$
\sigma_{i j}=C_{i j k l} \varepsilon_{k l}
$$

in which $C_{i j k l}$ is the fourth-order isotropic secant stiffness tensor, expressed as:

$$
C_{i j k l}=2 G \delta_{i k} \delta_{j l}+\left(K-\frac{2}{3} G \delta_{i j} \delta_{k l}\right)
$$

where $G=E /[2(1+v)]$ and $K=E /[3(1-2 v)]$ are the shear and bulk moduli, respectively, depending only on the stiffness $E$ and Poisson's ratio $v$. Fiber and matrix damage is modeled using a continuum damage mechanics (CDM) model proposed in [25,41-43], in which the elastic stiffness reduces with increasing deformation-induced damage. Elastic energy equivalence assumes that the elastic complimentary energy in a damaged material 
with the actual stress is equal to that in a hypothetical material with a fictitious effective stress. This establishes a relation between the damaged and undamaged elastic stiffnesses for an isotropic material as:

$$
E=(1-D)^{2} E^{0}
$$

where $D$ is a scalar damage variable, and $E$ and $E^{0}$ are elastic modulus in the damaged and undamaged state, respectively. A strain-rate and temperature-dependent expression for stiffness $E^{0}$ is obtained from [44] as:

$$
E_{\text {local }}^{0}=E_{\text {ref }}\left(1+C \ln \frac{\dot{\bar{\varepsilon}}}{\dot{\varepsilon_{0}}}\right)+\vartheta\left(\frac{T}{T_{0}}\right)
$$

where $E_{\text {local }}^{0}$ is local initial stiffness which is a function of the equivalent strain-rate $\dot{\varepsilon}$ and temperature $T . E_{\text {ref }}$ is stiffness at a reference strain-rate $\dot{\varepsilon_{0}}$, which is taken as $\dot{\varepsilon_{0}}=1 \mathrm{~s}^{-1}$. The function $\vartheta$ is chosen such that $\vartheta\left(\frac{T}{T_{0}}\right)=0$ for $T_{0}=22^{\circ} \mathrm{C}$. For the brittle materials, the damage variable is assumed to evolve following a damage evolution law [45] as:

$$
\dot{D}= \begin{cases}\left(\bar{\varepsilon} / \varepsilon_{0}\right)^{S^{*} \dot{\bar{\varepsilon}}} & \text { when } \bar{\varepsilon} \geq \varepsilon_{D} \text { and } \dot{\bar{\varepsilon}}>0 \\ 0 & \text { when } \bar{\varepsilon}<\varepsilon_{D} \text { or } \dot{\bar{\varepsilon}} \leq 0\end{cases}
$$

$\varepsilon_{D}$ is threshold effective strain for damage nucleation, exponent $S^{*}$ is a shape factor of the damage function, and $\bar{\varepsilon}=\sqrt{\frac{2}{3} \varepsilon_{i j} \varepsilon_{i j}}$ is the equivalent strain. The first in Equation (19) indicates that the damage increases when the equivalent strain is at or beyond the damage surface and the rate of equivalent strain is positive. Thus, the elastic modulus degrades during loading. Unloading is characterized by the second in Equation (19), when the equivalent strain is inside the damage surface and the strain-rate is nonpositive. In this case, there is no change in damage or elastic modulus. For complete damage, the stress reduces to zero, indicating loss of load carrying capacity. The damage variable in the constitutive law makes the finite element solutions mesh-dependent. To avert this shortcoming, a nonlocal damage formulation is incorporated in this model following [46], where a nonlocal damage parameter is expressed as:

$$
\dot{D}_{n}(\boldsymbol{x})=\frac{1}{V_{r}(\boldsymbol{x})} \int_{V} \alpha(\boldsymbol{s}-\boldsymbol{x}) \dot{D}(\boldsymbol{s}) d V(\boldsymbol{s})
$$

where

$$
V_{r}(\boldsymbol{x})=\int_{V} \alpha(\boldsymbol{s}-\boldsymbol{x}) d V(\boldsymbol{s}), \alpha(\boldsymbol{x})=\exp \left[-\left(\frac{\kappa|\boldsymbol{x}|}{l_{c}}\right)^{2}\right]
$$

$\kappa$ is a constant parameter and $l_{c}$ is a characteristic length that is of the same order of magnitude as the maximum size of inhomogeneities [46]. In these simulations, a value $l_{c}=8 \mu \mathrm{m}$ is taken.

\section{Cohesive zone model for interfacial debonding}

A 3D micromechanical model for composite microstructures undergoing fiber-matrix interfacial debonding has been developed in [47]. In this model, the fiber-matrix interface behavior in the normal and tangential directions is described by a nonlinear 3D cohesive zone model with bilinear traction-displacement relations. The interface is represented by a set of cohesive springs of infinitesimal length that are attached to the fiber and the matrix at opposite ends. With increasing displacement the traction across the interface reaches a maximum value, then decreases with further displacement increase, and finally 
vanishes indicating failure of the spring. Ortiz and co-workers [48,49] have developed irreversible cohesive laws for the unloading path after the interfacial softening.

In the present cohesive zone model, the relation between traction $T(=$ $\left.\sqrt{T_{n}^{2}+T_{t 1}^{2}+T_{t 2}^{2}}\right)$ and the effective opening displacement $\delta\left(=\left\|\mathbf{u}_{\mathbf{m}}-\mathbf{u}_{\mathbf{f}}\right\|\right)$ is given in terms of a free energy potential as

$$
T=\sqrt{T_{n}^{2}+T_{t 1}^{2}+T_{t 2}^{2}}=\frac{\partial \phi\left(\delta_{n}, \delta_{t 1}, \delta_{t 2}, q\right)}{\partial \delta}
$$

where $\left(T_{n}, T_{t 1}, T_{t 2}\right)$ are normal and tangential components of the interfacial traction and $q$ are internal variables that account for the inelastic process of decohesion. The effective opening displacement jump, $\delta$, in $3 \mathrm{D}$ is defined as

$$
\delta=\sqrt{\delta_{n}^{2}+\beta^{2} \delta_{t}^{2}}
$$

Here, $\delta_{n}$ is the displacement jump in normal direction, and $\delta_{t}\left(=\sqrt{\delta_{t_{1}}^{2}+\delta_{t_{2}}^{2}}\right)$ is the net displacement jump in tangential direction across the interface. $\beta$ is a factor that controls the contribution of the tangential component to the effective displacement jump. The scalar form of the $T-\delta$ relation in the bilinear model is obtained from Equation (22) as

$$
T= \begin{cases}\frac{\sigma_{\max }}{\delta_{c}} \delta & \text { if } \delta \leq \delta_{c} \quad \text { (hardening region) } \\ \frac{\sigma_{\max }}{\delta_{c}-\delta_{e}}\left(\delta-\delta_{e}\right) & \text { if } \delta_{c}<\delta \leq \delta_{e} \quad \text { (softening region) } \\ 0 & \text { if } \delta>\delta_{e} \quad \text { (complete debonding) }\end{cases}
$$

Consequently, the normal and tangential tractions are derived from the relations:

$$
T_{n}=\frac{\partial \phi}{\partial \delta_{n}}\left\{\begin{array}{ll}
\frac{\sigma_{\max }}{\delta_{c}} \delta_{n} & \text { if } \delta \leq \delta_{c} \\
\frac{\sigma_{\max }}{\delta} \frac{\delta-\delta_{e}}{\delta_{c}-\delta_{e}} \delta_{n} & \text { if } \delta_{c}<\delta \leq \delta_{e} \\
0 & \text { if } \delta>\delta_{e}
\end{array} \quad(i=1,2)\right.
$$

and

$$
T_{t i}=\frac{\partial \phi}{\partial \delta_{t i}}\left\{\begin{array}{ll}
\frac{\sigma_{\max }}{\delta_{c}} \beta^{2} \delta_{t i} & \text { if } \delta \leq \delta_{c} \\
\frac{\sigma_{\max }}{\delta} \frac{\delta-\delta_{e}}{\delta_{c}-\delta_{e}} \beta^{2} \delta_{t i} & \text { if } \delta_{c}<\delta \leq \delta_{e} \\
0 & \text { if } \delta>\delta_{e}
\end{array} \quad(i=1,2)\right.
$$

For a positive normal displacement $\delta_{n}$, the traction at the interface increases linearly to a maximum value of max $\sigma_{\max }$ corresponding to $\delta_{c}$. After that, the traction starts decreasing with increasing separation and finally reaches zero at a value of $\delta_{e}$. The unloading behavior in the hardening region follows the same slope as that of the loading path. In the softening region, unloading is assumed to follow a different linear path back from the current position to the origin with a reduced stiffness. This is expressed as

$$
T=\frac{\sigma_{\max }}{\delta_{\max }} \frac{\delta_{\max }-\delta_{e}}{\delta_{c}-\delta_{e}} \delta \quad \delta_{c}<\delta_{\max }<\delta_{e} \text { and } \delta<\delta_{\max }
$$

Reloading follows the unloading slope till it meets the point of unloading in the softening plot and then continues along the softening plot. This demonstrates the irreversible nature of the damage process. The normal component of the traction is transferred through the normal springs, while the shear component of the traction is transferred through the tangential springs. Both the normal and the tangential tractions vanish when interface debonds completely, i.e., $\delta \geq \delta_{e}$. Also, the magnitudes of the tangential tractiondisplacement relation are independent of the sign, and hence, the behavior is the same for both positive and negative tangential separations $\delta_{i 1}$ and $\delta_{i 2}$. If the normal displacement is negative, i.e., during compression, stiff penalty springs with high stiffness are introduced 


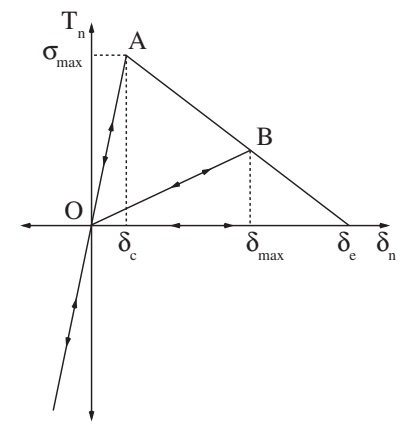

(a)

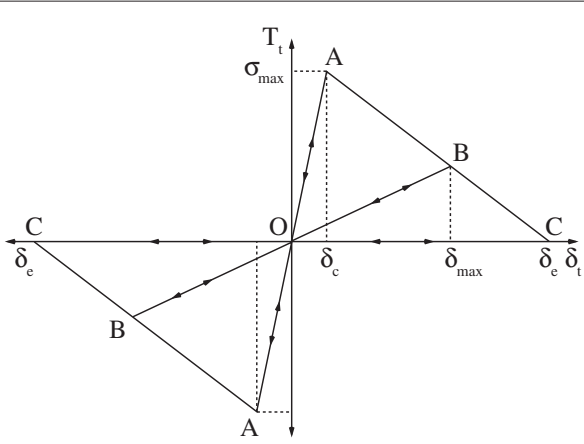

(b)

Figure 11 Normal (a) and tangential (b) traction-displacement behavior for a bilinear cohesive zone model.

between the node-pairs at the interface to prevent penetration. Figure 11a,b shows the normal traction-separation response for $\delta_{t}=0$ and the tangential traction-separation response for $\delta_{n}=0$, respectively.

\section{Micromechanical analysis of a SERVE undergoing interfacial debonding and fiber/matrix} damage

Micromechanical analyses of multi-fiber RVEs are conducted under uniaxial tension to study fiber and matrix failure and interface debonding at different strain-rates. Interaction of the two damage modes leads to crack propagation in the RVE. All simulations are conducted using the trilinear eight-noded brick element with reduced integration (C3D8R) in ABAQUS-Explicit. The nonlocal continuum damage model is implemented in the user subroutine VUMAT, while the bilinear cohesive model is implemented in the VUEL user subroutine. The material and damage properties, as well as cohesive zone parameters used in this analysis, are listed in Table 2. The material properties include Young's modulus, Poisson's ratio and density. The calibration and validation of these parameters will be reported in another paper by the author's group shortly.

To examine the convergence characteristics of the finite element mesh for problems involving damage, a RVE corresponding to a hexagonal array of fibers is analyzed using four different meshes. The size of the RVE is $2.0 \mathrm{~mm} \times 2.0 \mathrm{~mm} \times 1.0 \mathrm{~mm}$ with a fiber volume fraction of $10 \%$. The coarsest mesh is composed of 9,568 brick elements, while the finest mesh is composed of 220,160 elements. A uniaxial tensile strain-rate $10^{2} s^{-1}$ is applied to the RVE. Figure 12a shows the contour of damage variable $D$ in the microstructure at $0.05 \mathrm{~s}$. The local strain-rates range between $10^{3} \mathrm{~s}^{-1}$ and $10^{7} \mathrm{~s}^{-1}$ and are much higher than the overall loading rate. Figure $12 \mathrm{~b}$ plots the ratio of the damage bandwidth $h_{D}$ in Figure $12 \mathrm{a}$ to the dimension of the RVE $L_{\mathrm{RVE}}$ with increasing mesh size. The figure

Table 2 Fiber and matrix properties and interface (cohesive zone) material properties

\begin{tabular}{cccccccccc}
\hline Medium & $\begin{array}{c}\boldsymbol{E}^{\mathbf{0}} \\
(\mathrm{GPa})\end{array}$ & $\boldsymbol{v}$ & $\begin{array}{c}\boldsymbol{\rho} \\
\left(\mathbf{k g} / \mathbf{m}^{\mathbf{3}}\right)\end{array}$ & $\boldsymbol{\epsilon}^{\boldsymbol{D}}$ & $\boldsymbol{\epsilon}^{\mathbf{0}}$ & $\boldsymbol{S}^{*}$ & $\begin{array}{c}\boldsymbol{\sigma}_{\max } \\
(\mathrm{MPa})\end{array}$ & $\begin{array}{c}\boldsymbol{\delta}_{\boldsymbol{e}} \\
(\mu \mathbf{s})\end{array}$ & $\begin{array}{c}\sigma_{\max } / \delta_{\boldsymbol{c}} \\
(\mathrm{N} / \mathbf{m})\end{array}$ \\
\hline Fiber (f) & 64 & 0.26 & 2,230 & 0.0164 & 0.001 & 7 & & & \\
Matrix (m) & 2.5 & 0.40 & 1,170 & 1.50 & 0.001 & 7 & & & \\
Interface (CZM) & & & & & & & 4.0 & 5 & 20,000 \\
\hline
\end{tabular}


(a)

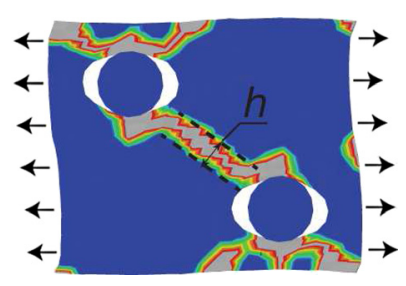

(b)

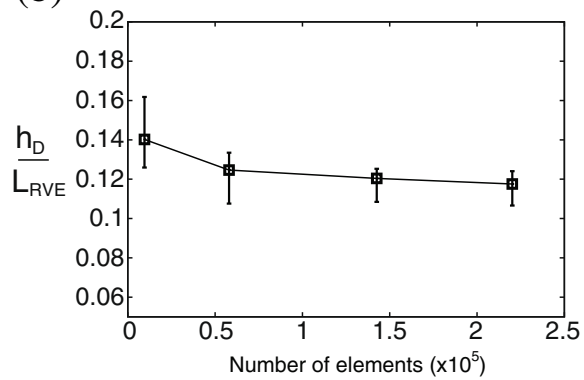

Figure 12 Contour plot of damage in the RVE. (a) Contour plot of damage in the RVE showing the width of the damage band, and (b) plot showing mesh sensitivity of nonlocal CDM model.

shows the average value of $\frac{h_{D}}{L_{\mathrm{RVE}}}$ along with the variation bars of the damage band thickness. Results in Figure 12b suggest that the solution tends to converge with the higher mesh densities.

Figure 13 shows the progression of damage for a RVE model with 172 fibers, with a uniform fiber radius of $2 \mu \mathrm{m}$ and fiber volume fraction of $21.6 \%$. The figures are plotted at different times during the tensile loading process in the horizontal direction. Damage nucleates with interfacial debonding and subsequently grows as matrix damage ahead of the interfacial cohesive zone crack tip, where stresses are large. In the progressive matrix crack growth, the crack tends to grow to the nearest debonded interface tip or the nearest matrix crack. Figure 13d shows that parallel cracks do not tend to coalesce, whereas nonparallel cracks are likely to merge together into one long but zigzag crack.
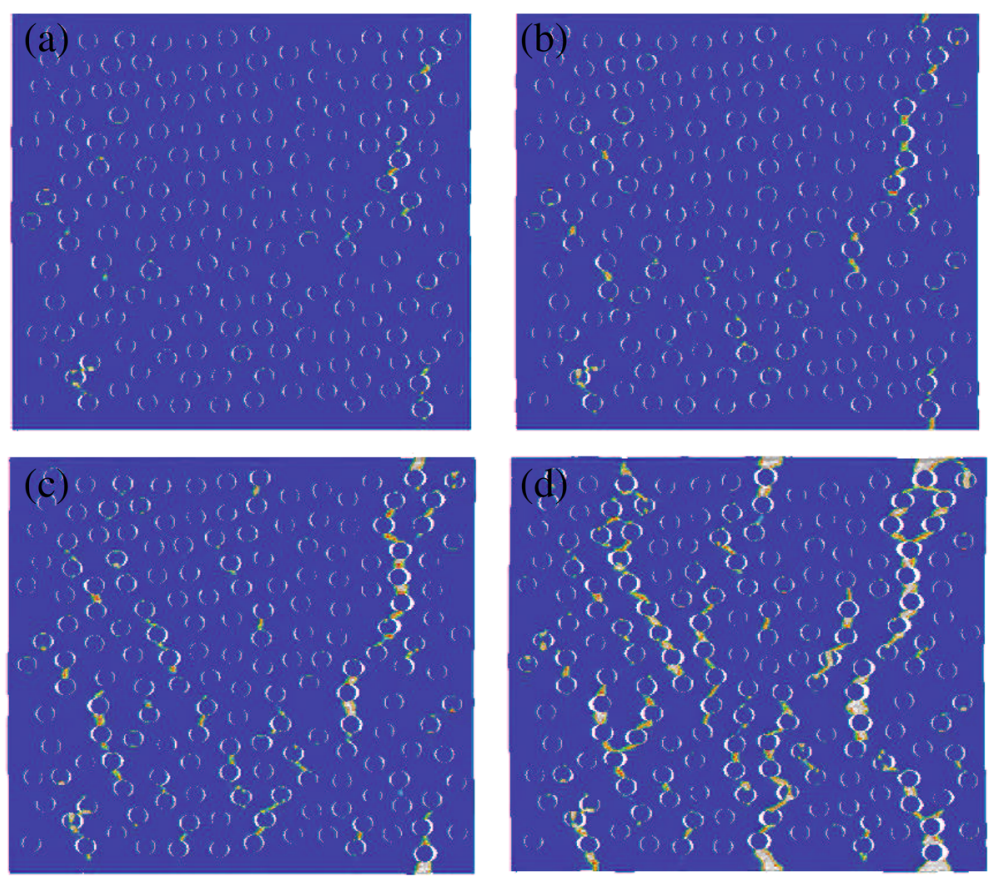

Figure 13 Damage evolution in a RVE with 172 randomly located fibers. At (a) $t=0.01 \mu \mathrm{s}$, (b) $t=0.011 \mu \mathrm{s}$, (c) $t=0.012 \mu \mathrm{s}$, and (d) $t=0.015 \mu \mathrm{s}$. 


\section{Homogenization-based continuum damage mechanics model}

For predicting damage in composites at the macroscales, Ghosh and coworkers have developed anisotropic HCDM models in [25-27]. This model can avoid the need to perform micromechanical analysis at each load increment and is computationally very efficient. The general form of CDM models [41] introduces a fictitious stress $\tilde{\Sigma}_{i j}$ acting on an effective resisting area $(\tilde{A})$, which is caused by reduction of the original resisting area $A$ due to material degradation from the presence of microcracks and stress concentration in the vicinity of cracks. In $[11,42]$, the effective stress $\tilde{\Sigma}_{i j}$ is related to the actual Cauchy stress $\Sigma_{i j}$ through a fourth-order damage effect tensor $M_{i j k l}$ as

$$
\tilde{\Sigma}_{i j}=M_{i j k l}(\mathbf{D}) \Sigma_{k l}
$$

where $M_{i j k l}$ is a function of a damage tensor $\mathbf{D}\left(=D_{i j k l} \mathbf{e}_{\mathbf{i}} \otimes \mathbf{e}_{\mathbf{j}} \otimes \mathbf{e}_{\mathbf{k}} \otimes \mathbf{e}_{\mathbf{l}}\right)$. D can be a zeroth-, second-, or fourth-order tensor, depending on the model employed. The hypothesis of equivalent elastic energy is used to evaluate $M_{i j k l}$ and establish a relation between the damaged and undamaged stiffnesses [50,51]. The hypothesis, detailed in $[52,53]$, specifically assumes that the elastic complimentary energy $W_{C}$ in a damaged material with the actual stress is equal to that in a hypothetical undamaged material with the fictitious effective stress, i.e.,

$$
W_{C}(\mathbf{\Sigma}, \mathbf{D})=\frac{1}{2}\left(C_{i j k l}(\mathbf{D})\right)^{-1} \Sigma_{i j} \Sigma_{k l}=W_{C}(\tilde{\boldsymbol{\Sigma}}, \mathbf{0})=\frac{1}{2}\left(C_{i j k l}^{o}\right)^{-1} \tilde{\Sigma}_{i j} \tilde{\Sigma}_{k l}
$$

where $\boldsymbol{\Sigma}=\Sigma_{i j} \mathbf{e}_{\mathbf{i}} \otimes \mathbf{e}_{\mathbf{j}}, E_{i j k l}^{o}$ is the elastic stiffness tensor in the undamaged state, and $E_{i j k l}(\mathbf{D})$ is the stiffness in a damaged state. From Equations (28) and (29), the relation between the damaged and undamaged stiffnesses is established as

$$
C_{i j k l}=\left(M_{p q i j}\right)^{-1} C_{p q r s}^{o}\left(M_{r s k l}\right)^{-T}
$$

where $-T$ corresponds to the transpose of the inverse of the fourth-order $\mathbf{M}$ tensor. With the choice of an appropriate order of the damage tensor and the assumption of a function for $M_{i j k l}$, Equation (30) can be used to formulate a damage evolution model using micromechanics and homogenization. The HCDM model assumes a diffused damage state and correspondingly a positive semi-definite stiffness matrix, even though the microstructural SERVE from which it is developed has regions of softening and damage localization.

The HCDM model proposed in [25-27] introduces a damage evolution surface to delineate the interface between damaged and undamaged domains in the strain $\left(e_{i j}\right)$-space as

$$
F=\frac{1}{2} P_{i j k l} e_{i j} e_{k l}-\kappa\left(\alpha W_{d}\right)=0
$$

where $W_{d}$ is the dissipation of the strain energy density due to stiffness degradation expressed as:

$$
W_{d}=\int \frac{1}{2} e_{i j} e_{k l} d C_{i j k l}
$$

Assuming associativity rule in the stiffness space, the evolution of the fourth-order secant stiffness is obtained as

$$
\dot{C}_{i j k l}=\dot{\lambda} \frac{\partial F}{\partial\left(\frac{1}{2} e_{i j} e_{k l}\right)}=\dot{\lambda} P_{i j k l}
$$


$P_{i j k l}$ is a fourth-order symmetric negative definite tensor that corresponds to the direction of the rate of stiffness degradation tensor $\dot{C}_{i j k l} . P_{i j k l}$ is expressed as a function of strain $e_{i j}$, $\alpha$ is a scaling parameter, and $\kappa$ is a function of $W_{d}$.

HCDM model in the principal damage coordinate system

For a second-order damage tensor $D_{i j}$, the damage effect tensor $M_{i j k l}$ in Equation (28) has been defined in [54] as:

$$
M_{i j k l}=\left(\delta_{i k}-D_{i k}\right)^{-1} \delta_{j l}
$$

It has been shown in [54] that $D_{i j}$ is symmetric and it can describe the damage states which have at least orthotropic symmetry. An implicit method of rendering the stress tensor symmetric has been suggested in [55], which corresponds to a representation of the stress tensor in a fixed global coordinate system as

$$
\Sigma_{i j}=\frac{\tilde{\Sigma}_{i k}\left(\delta_{k j}-D_{k j}\right)^{-1}+\left(\delta_{i l}-D_{i l}\right)^{-1} \tilde{\Sigma}_{l j}}{2}
$$

The corresponding inverse of the damage effect tensor $\left[\mathbf{M}\left(D_{i j}\right)\right]^{-1}$ is represented in a matrix form as:

$$
\left[\mathbf{M}\left(D_{i j}\right)\right]^{-1}=\left[\begin{array}{lccccc}
1-D_{11} & 0 & 0 & 0 & -D_{13} & -D_{12} \\
0 & 1-D_{22} & 0 & -D_{23} & 0 & -D_{12} \\
0 & 0 & 1-D_{33} & -D_{23} & -D_{13} & 0 \\
0 & -\frac{1}{2} D_{23} & -\frac{1}{2} D_{23} & 1-\frac{1}{2}\left(D_{22}+D_{33}\right) & -D_{12} & -D_{13} \\
-\frac{1}{2} D_{13} & 0 & -\frac{1}{2} D_{13} & -\frac{1}{2} D_{12} & 1-\frac{1}{2}\left(D_{11}+D_{33}\right) & -D_{23} \\
-\frac{1}{2} D_{12} & -\frac{1}{2} D_{12} & 0 & -\frac{1}{2} D_{13} & -\frac{1}{2} D_{23} & 1-\frac{1}{2}\left(D_{11}+D_{22}\right)
\end{array}\right]
$$

This can be substituted in Equation (30) to update the damaged stiffness $C_{i j k l}$ from the initial undamaged stiffness $C_{i j k l}^{o}$.

Numerical examples in [11] have shown that material symmetry is considerably affected by damage evolution in composite microstructures. Different load paths will yield different damage profiles in the microstructure, and this will alter the initial material symmetry in $C_{i j k l}^{o}$ in different ways. In a fixed coordinate system, an RVE exhibiting orthotropy in $C_{i j k l}^{o}$ can exhibit general anisotropy with evolving damage under multi-axial loading. In the fixed coordinate system, the anisotropic $C_{i j k l}$ will couple normal and shear strain components in the elastic energy expression. However, when the strains are represented in a coordinate system that corresponds to the principal damage axes, the coupling terms in the stiffness $C_{i j k l}$ reduce to near vanishing values and the initial symmetry properties are retained. Hence, the homogenized stiffness matrix has been represented in the PDCS in [25-27]. Determination of the continuously evolving principal damage coordinate system requires the determination of the second-order damage tensor $D_{i j}$ and subsequent evaluation of its eigen-vectors at each step of the incremental loading process. A transformation matrix $[\mathbf{Q}]^{D}$ is formed from the eigen-vectors of $D_{i j}$, which leads to the rotation of the global coordinate system to the principal damage coordinate system.

An issue with the HCDM formulation in [25-27] is that the coefficients $P_{i j k l}$ have been derived in terms of the applied strain to the RVE. This makes them dependent on the external load, which leads to a load-dependent (rather than material state-dependent) damage relation. To overcome this, a new formulation is proposed in this work. 


\section{Damage evolution laws in the PDCS}

The macroscopic damage evolution surface is rewritten in the PDCS as:

$$
F^{\prime}=\frac{1}{2} Y_{i j}^{\prime} P_{i j k l}^{\prime} Y_{k l}^{\prime}-1=0
$$

where $Y_{i j}^{\prime}$ is thermodynamic force conjugate of the damage tensor $D_{i j}^{\prime}$ given as:

$$
Y_{i j}^{\prime}=-\frac{1}{2} e_{p q}^{\prime} \frac{\partial C_{p q r s}^{\prime}}{\partial D_{i j}^{\prime}} e_{r s}^{\prime}
$$

The prime in the superscript denotes quantities expressed in the PDCS using the transformation laws. The fourth-order tensor $P_{i j k l}^{\prime}$ evolves with initiation and anisotropic growth of the damage. Assuming associativity in the damage space, the damage evolution rate is given in [56] as:

$$
\dot{D}_{i j}^{\prime}=\dot{\lambda} \frac{\partial F^{\prime}}{\partial Y_{i j}^{\prime}}=\dot{\lambda} P_{i j k l}^{\prime} Y_{k l}^{\prime}
$$

The Clausius-Duhem inequality and the Kuhn Tucker condition for the irreversible nature of the damage are respectively defined as:

$$
\begin{aligned}
& \dot{D_{i j}^{\prime}} Y_{i j}^{\prime} \geq 0 \\
& F^{\prime} \leq 0, \dot{\lambda} \geq 0, \dot{\lambda} F^{\prime}=0
\end{aligned}
$$

The proposed HCDM model requires the calibration of $P_{i j k l}$ from the micromechanical analysis of RVE. The fourth-order tensor $P_{i j k l}$ in the HCDM model is a function of the strain energy dissipation due to damage $W_{d}$ that is calculated in the PDCS as

$$
W_{d}=\frac{1}{2} \int e_{i j}^{\prime} e_{k l}^{\prime} d C_{i j k l}^{\prime}
$$

\section{Evaluation of $P_{i j k l}^{\prime}$ from micromechanical analysis of the RVE}

In the incremental finite element formulation for damage evolution, the backward Euler method is used to integrate the rate of damage evolution in Equation (38). For a strain increment for step $n$ to $n+1, P_{i j k l}$ is given by

$$
\left(P_{i j k l}^{\prime}\right)_{n+1}\left(Y_{k l}^{\prime}\right)_{n+1}=\frac{\left(D_{i j}^{\prime}\right)_{n+1}-\left(D_{i j}^{\prime}\right)_{n}}{\lambda_{n+1}-\lambda_{n}}
$$

Substituting this equation in Equation (36), the parameter $\lambda_{n+1}$ is evaluated from the relation:

$$
\lambda_{n+1}=\lambda_{n}+\frac{1}{2}\left(\left(D_{i j}^{\prime}\right)_{n+1}-\left(D_{i j}^{\prime}\right)_{n}\right)\left(Y_{i j}^{\prime}\right)_{n+1}
$$

The coefficients $\left(P_{i j k l}\right)_{n+1}$ are subsequently determined using Equation (42) from the homogenized response of the micromechanical analysis. The components $P_{i j k l}$ obtained from micromechanical analysis can be represented as a function of the evolving variable $W_{d}$ using radial basis functions (RBFs). They can be approximated by the linear combination of radially symmetric RBFs [57] as:

$$
P_{i j k l}\left(W_{d}\right)=\sum_{\alpha=1}^{M}\left(a_{i j k l}\right)_{\alpha} \phi\left(\frac{\left\|W_{d}-\left(W_{d}\right)_{\alpha}\right\|}{b_{\alpha}}\right)
$$


where $M$ is the number of kernel nodes, $\left(a_{i j k l}\right)_{\alpha}$ are the tensor weighting coefficients and $\phi$ is a Gaussian radial basis function given by

$$
\phi(r)=\exp \left(-r^{2}\right)
$$

and $\left(W_{d}\right)_{\alpha}$ and $b_{\alpha}$ are the centroid and smoothing factor or width of the $\alpha$-th kernel node, respectively. In Equation (44), $\|\cdots\|$ denotes the Euclidean distance and $\left(W_{d}\right)_{\alpha}$ are the reference points chosen from the micromechanical data. The weighting coefficients $\left(a_{i j k l}\right)_{\alpha}$ are evaluated from micromechanical analysis using the procedure outlined in [57]. The proposed HCDM model is implemented in ABAQUS using the user subroutine UMAT as discussed in [25-27]. The comparison of $P_{i j k l}\left(W_{d}\right)$ from micromechanical results and the calibrated expression using RBFs is shown in Figure 14. The HMM model is obtained by volume averaging the micromechanical stresses and strains in the RVE, subjected to periodic boundary conditions with an imposed macroscopic strain. The HCDM model parameter is able to capture the variations in the micromechanical analysis with excellent agreement.

\section{Macro-micro analysis of a composite structure with the HCDM model}

This section demonstrates the strength of the HCDM model as an analysis tool for composite applications by establishing a connection between macroscopic damage evolution and explicit microscopic failure mechanisms. Structural simulations of damage evolution are conducted by the code ABAQUS with the HCDM model incorporated in the user subroutine UMAT. Without any loss of generality, fiber-matrix interface debonding is taken as the microstructural damage mechanism in this study. The macroscopic finite element model implementing the HCDM constitutive relations consists of eightnoded quadrilateral elements. Four three-dimensional fiber-matrix composite RVEs with different morphological arrangements are considered for this validation study. These are:

(a) Unidirectional 3D uniform composite microstructure with fibers arranged in a rectangular array. The RVE is a unit cell containing a single cylindrical fiber of volume fraction $20 \%$.

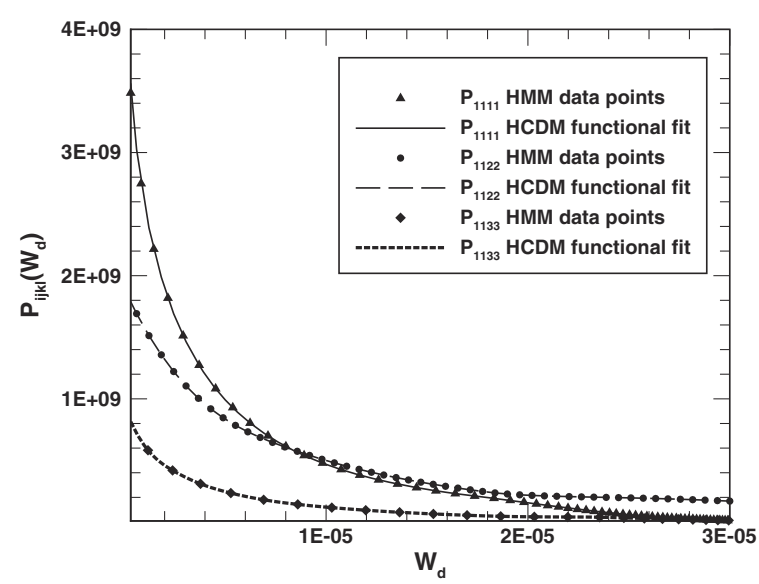

Figure 14 Comparing RBF-based functional representation of $P_{i j k l}\left(W_{d}\right)$ with from the homogenized micromechanics (HMM) data. 
(b) Unidirectional 3D uniform composite microstructure with fibers arranged in a rectangular array. The RVE is a unit cell containing a single fiber of elliptical cross section. Volume fraction is $20 \%$, and the aspect ratio of the elliptical cross section is $\frac{a}{b}=2$.

(c) Unidirectional 3D composite microstructure with hexagonal arrangement of fibers. The RVE contains cylindrical fibers with a fiber volume fraction is $20 \%$.

(d) Cross-ply 3D composite microstructure with its RVE consisting of two cylindrical fibers oriented at $90^{\circ}$ with respect to each other. The fiber volume fraction in the RVE is $20 \%$.

The simulations involve a deformable composite projectile impactor colliding with a rigid surface. The impactors, consisting of one of the four microstructural architectures, is macroscopically cylindrical in shape with radius of $3.2 \mathrm{~mm}$ and length of $32.4 \mathrm{~mm}$. It moves with an initial velocity of $10 \mathrm{~m} / \mathrm{s}$. The density of epoxy matrix is assumed to be 750 $\mathrm{kg} / \mathrm{m}^{3}$ while that of steel fiber is $7,800 \mathrm{~kg} / \mathrm{m}^{3}$ so that, for fiber volume fraction of $20 \%$, the density of the aggregate is $2,160 \mathrm{~kg} / \mathrm{m}^{3}$. The rigid surface and the projectile are modeled using continuum elements, and contact between projectile and rigid surface is assumed to be frictionless.

The total energy dissipated due to evolving damage $W_{d}$ is plotted as a function of time in Figure 15 for the different composite microstructures. The composite impactor with RVE (a) undergoes maximum amount of damage while that with the cross-ply RVE (d) undergoes only nominal damage. For the composite with elliptical fiber reinforced RVE (b), the damage initiates earlier than that for RVEs (a) and (c). However, it accumulates at a lower rate, resulting in significantly lower value of $W_{d}$ at the end of the simulation. Figure 16a,b,c,d) show contour plots of energy dissipated $W_{d}$ due to evolving damage at $5.0 \mu \mathrm{s}$ for the different microstructures. The damage initiates near the periphery of the impactor head and then propagates towards the center of the head cross-section. Different loading states affect the evolution of damage in each microstructural configuration in different ways resulting in different damaged behaviors at various locations in the structure. However, the distribution of $W_{d}$ is more uniform in composite with RVE (a), and

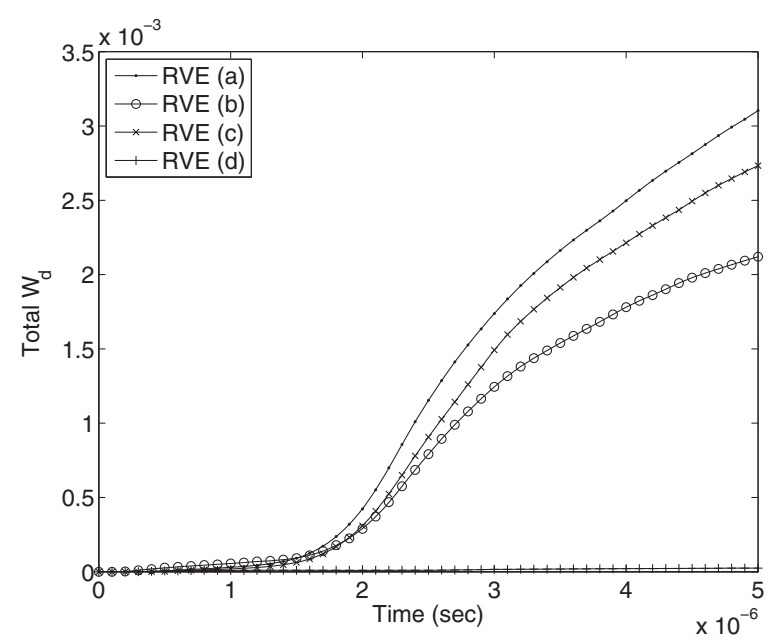

Figure 15 Total energy dissipated $\left(W_{d}\right)$ due to evolving damage in the composite impactor for four different microstructures. 


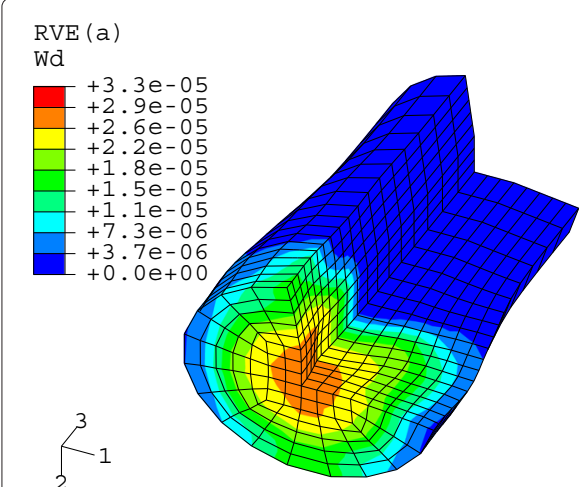

(a)

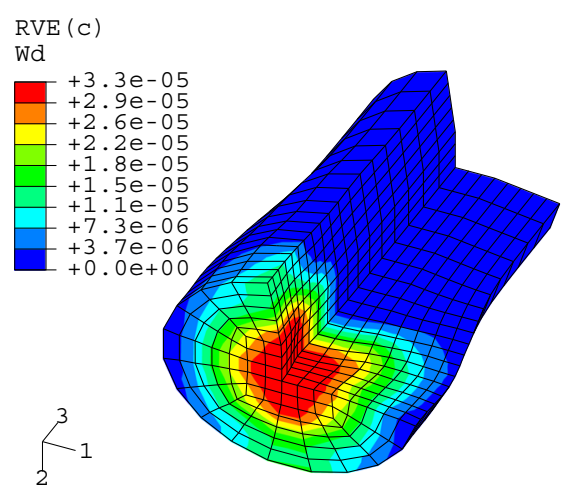

(c)

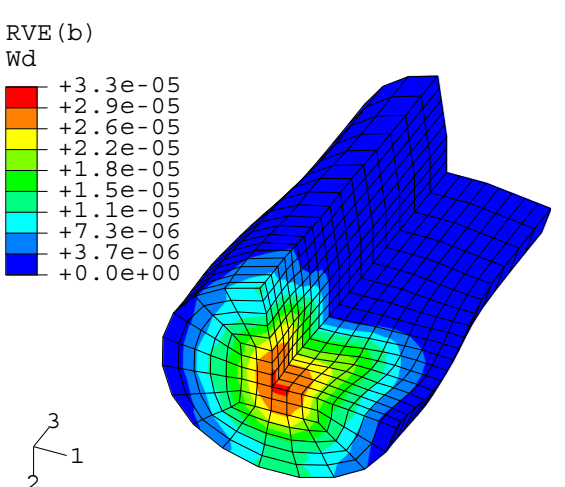

(b)

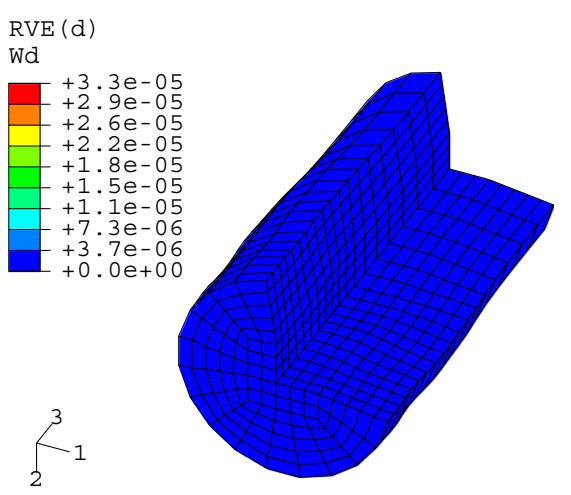

(d)

Figure 16 Contour plots of dissipated energy $\left(W_{d}\right)$ due to damage with RVEs (a), (b), (c), and (d) at time = $5.0 \mu \mathrm{s}$.

hence, the total $W_{d}$ is more than for the other RVEs. The homogenization method allows for the assessment of stress-strain and damage evolution in the microstructural RVE, subject to a given macroscopic strain history. Figure 17a,b,c,d shows the stress $\sigma_{11}$ contour plots in the microstructural RVEs at a point on the face of the impactor.

\section{Conclusions}

This paper provides an ICMSE perspective on some of the aspects, governing multiscale modeling of composite materials. While a rigorous ICMSE framework encompasses several ingredients, only a partial list is dealt with in this paper. The major items discussed include microstructure characterization and statistically equivalent representative volume elements or SERVE identification, micromechanical analyses of the SERVE, and development of homogenized models by hierarchical modeling.

Alternative methods of identifying SERVEs using both morphology- and micromechanics-based methods are proposed for undamaged and damaging conditions. Similar sizes of the SERVE are predicted by these alternate methods, suggesting that the different indicators are able to predict similar influence of microstructural elements on one another. An important contribution to the identification of the SERVE is the development of boundary conditions based on the statistical distribution of heterogeneities in 


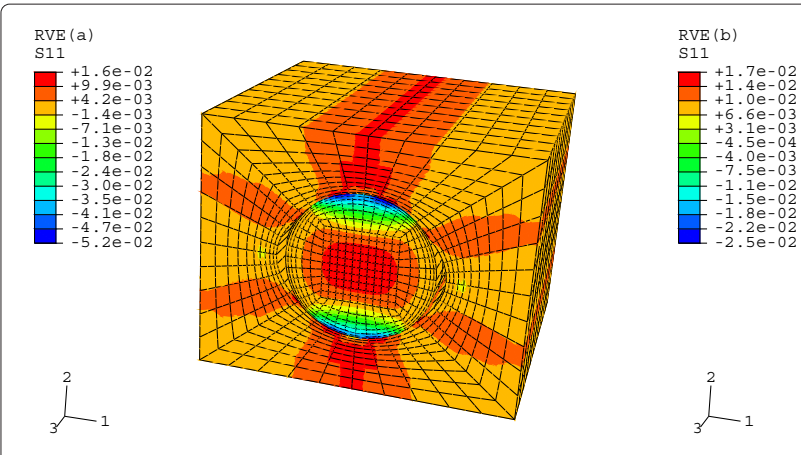

(a)

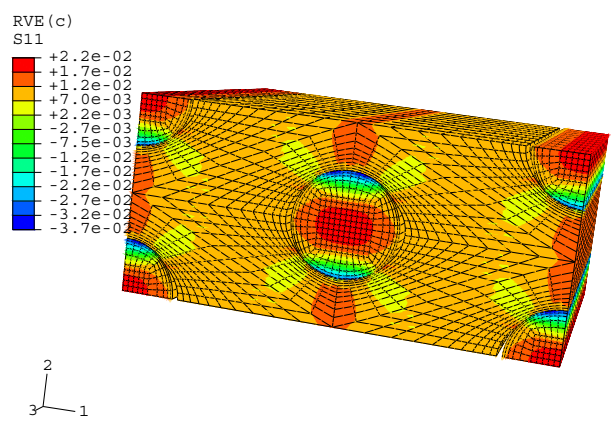

(c)

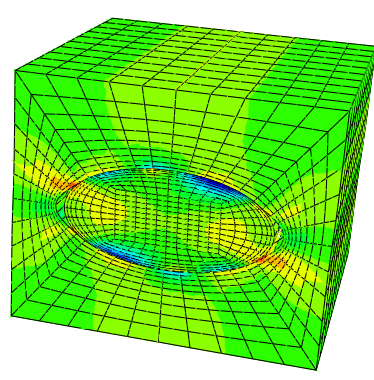

(b)
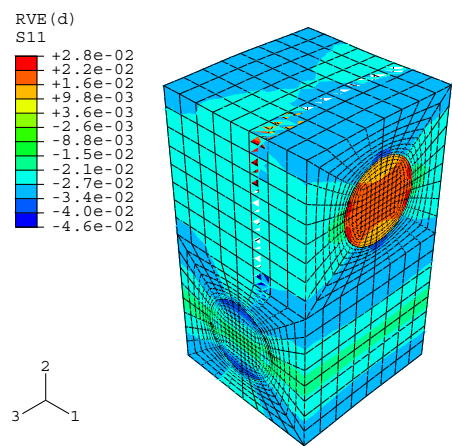

(d)

Figure 17 Contour plots of microscopic stress $\left(\sigma_{11}\right)$ at the head of composite impactor for RVE's (a), (b), (c), and (d).

the domain exterior to the SERVE. In this formulation, the two-point correlation function is combined with the Green's function approach to derive accurate displacement boundary conditions on the SERVE. The SERVE continuously evolves in size with the evolution of microscopic damage. It is expected that the imposition of accurate boundary conditions will reduce the size of the SERVE that is conventionally needed with periodic, uniform traction, or displacement boundary conditions.

A micromechanical analysis model of the composite microstructural SERVE, undergoing damage evolution in the form of fiber and matrix cracking and interfacial debonding, is developed. Interfacial debonding is modeled using a bilinear cohesive zone model, while nonlocal continuum damage mechanics models are used to represent fiber and matrix damage. Three-dimensional microstructures are simulated for crack propagation with this model.

Finally, an accurate and computationally efficient 3D HCDM is developed for composites undergoing interfacial debonding. The HCDM model represents orthotropic behavior in the PDCS and uses a fourth-order damage tensor which characterizes the stiffness as an internal variable. The model is found to accurately predict damage behavior for a wide range of proportional and nonproportional loading. The HCDM model-based simulations are able to provide both macroscopic and microscopic information on evolving damage and failure. The capability of macroscopic damage predictions in structures 
with explicit reference to the microstructural composition is largely lacking in the literature. The overall model framework presented in this study can be used in material design to enhance structural performance and life.

\section{Competing interests}

The authors declare that they have no competing interests.

\section{Acknowledgements}

This author acknowledges the contributions of his research group members Dr. D. Kubair, Ms. Z. Li, and Mr. S. Baby to the paper. This work has been partially supported through a grant No. FA9550-12-1-0445 to the Center of Excellence on Integrated Materials Modeling (CEIMM) at Johns Hopkins University awarded by the AFOSR/RSL Computational Mathematics Program (Manager Dr. F. Fahroo) and AFRL/RX (Monitors Drs. C. Woodward and C. Przybyla). It is also partially sponsored by the Center for Materials in Extreme Dynamic Environments (CMEDE) of the Army Research Laboratory and was accomplished under Cooperative Agreement Number W911NF-12-2-0022. These sponsorships are gratefully acknowledged.

Received: 27 November 2014 Accepted: 9 April 2015

Published online: 11 June 2015

\section{References}

1. Mura T (1987) Micromechanics of defects in solids. 2nd edn. Kluwer Academic Publishers and Martinus Nijhoff, Netherlands

2. Hashin Z (1970) Theory of composite materials. In: Wend FW, Leibowitz H, Perrone N (eds). Mechanics of Composite Materials. Pergamon Press, New York

3. Nemat-Nasser S, Yu N, Hori M (1993) Bounds and estimates of overall moduli of composites with periodic microstructure. Mech Mater 15:163-181

4. Budiansky B (1965) On the elastic moduli of some heterogeneous materials. J Mech Phys Sol 13:223-227

5. Christensen RM, Lo KH (1979) Solutions for effective shear properties in three phase sphere and cylinder models. J Mech Phys Sol 27:315-330

6. Tamma KK, Chung PW (1999) Woven fabric composites: developments in engineering bounds, homogenization and applications. Inter J Numer Meth Engng 45:1757-1790

7. Fish J, Shek K (2000) Multiscale analysis of composite materials and structures. Comp Sci Tech 60:2547-2556

8. Ghosh S, Lee K, Moorthy S (1995) Multiple scale analysis of heterogeneous elastic structures using homogenization theory and Voronoi cell finite element method. Int J Sol Struc 32(1):27-62

9. Ghosh S, Lee K, Moorthy S (1996) Two scale analysis of heterogeneous elastic-plastic materials with asymptotic homogenization and Voronoi cell finite element model. Comp Meth Appl Mech Engin 132(1-2):63-116

10. Guedes JM, Kikuchi N (1991) Preprocessing and postprocessing for materials based on the homogenization method with adaptive finite element methods. Comp Meth Appl Mech Engin 83:143-198

11. Raghavan P, Ghosh S (2005) A continuum damage mechanics model for unidirectional composites undergoing interfacial debonding. Mech Mater 37(9):955-979

12. Terada K, Kikuchi N (2000) Simulation of the multi-scale convergence in computational homogenization approaches. Int J Sol Struc 37:2285-2311

13. Feyel F, Chaboche JH (2000) FE $\mathrm{F}^{2}$ multiscale approach for modelling the elastoviscoplastic behaviour of long fibre $\mathrm{SiC} / \mathrm{Ti}$ composite materials. Comp Meth Appl Mech Engin 183:309-330

14. Ghosh S, Bai J, Paquet D (2009) Homogenization-based continuum plasticity-damage model for ductile failure of materials containing heterogeneities. J Mech Phys Sol 57(7):1017-1044

15. Fish J, Shek K (2000) Multiscale analysis of composite materials and structures. Comp Sci Tech 60:2547-2556

16. Oden JT, Zohdi TI (1997) Analysis and adaptive modeling of highly heterogeneous elastic structures. Comp Meth Appl Mech Engin 148:367-391

17. Oden JT, Vemaganti K, Moës N (1999) Hierarchical modeling of heterogeneous solids. Comp Meth Appl Mech Engin 172:3-25

18. Zohdi TI, Oden JT, Rodin GJ (1999) Hierarchical modeling of heterogeneous solids. Comp Meth Appl Mech Engin 172:3-25

19. Raghavan P, Ghosh S (2004) Concurrent multi-scale analysis of elastic composites by a multi-level computational model. Comp Meth Appl Mech Engin 193(6-8):497-538

20. Ghosh S (2008) Adaptive concurrent multi-level model for multi-scale analysis of composite materials including damage. In: Kwon Y, Allen DH, Talreja R (eds). Multiscale Modeling and Simulation of Composite Materials and Structures. Springer, New York. pp 83-164

21. Ghosh S (2015) Adaptive hierarchical-concurrent multi-scale modeling of ductile failure in heterogeneous metallic materials. JOM, J Miner Met Mater Soc 67(1):129-142

22. Ghosh S, Paquet D (2013) Adaptive multi-level model for multi-scale analysis of ductile fracture in heterogeneous aluminum alloys. Mech Mater 65:12-34

23. Kouznetsova V, Geers MGD, Brekelmans WAM (2002) Multi-scale constitutive modelling of heterogeneous materials with a gradient-enhanced computational homogenization scheme. Int J Numer Meth Engin 54:1235-1260

24. Vernerey FJ, Liu WK, Moran B, Olson G (2008) A micromorphic model for the multiple scale failure of heterogeneous materials. J Mech Phys Sol 56(4):1320-1347

25. Jain JR, Ghosh S (2008) Homogenization based 3d contiuum damage mechanics model for composites undergoing microstructural debonding. J Appl Mech 75:031011-15 
26. Jain JR, Ghosh S (2009) Damage evolution in composites with a homogenization based contiuum damage mechanics model. Int J Damage Mech 18(6):533-568

27. Ghosh S, Jain JR (2009) A homogenization based continuum damage mechanics model for cyclic damage in 3D composites. The Aero J 113(1144):371-383

28. Hill R (1963) Elastic properties of reinforced solids: some theoretical principles. J Mech Phys Sol 11:357-372

29. Hashin Z, Strikman S (1963) A variational approach to the theory of the elastic behavior of multiphase materials. J Mech Phys Sol 11:127-140

30. Jones RM (1975) Mechanics of composite materials. Hemisphere Publishing Corporation, USA

31. Drugan WJ, Willis JR (1996) A micromechanics-based non local constitutive equation and estimates of representative volume element size for elastic composites. J Mech Phys Sol 44:1497-524

32. Ghosh S, Swaminathan S, Pagano NJ (2006) Statistically equivalent representative volume elements for composite microstructures, part I: without damage. J Comp Mater 40(7):583-604

33. Swaminathann S, Ghosh S (2006) Statistically equivalent representative volume elements for composite microstructures, part II: with evolving damage. J Comp Mater 40(7):605-621

34. Ghosh S (2011) Micromechanical analysis and multi-scale modeling using the Voronoi cell finite element method. Florida, USA

35. Ghosh S, Lee K, Raghavan P (2001) A multi-level computational model for multiscale damage analysis in composite and porous materials. Int J Sol Struct 38(14):2335-2385

36. Pyrz R (1994) Quantitative description of the microstructure of composites. Part l: morphology of unidirectional composite systems. Comp Sci Techn 50:197-208

37. Pyrz R (1994) Correlation of microstructure variability and local stress field in two-phase materials. Mater Sci Engin A A177:253-259

38. Li S, Ghosh S (2004) Debonding in composite microstructures with morphologic variations. Int J Comp Meth 1(1):121-149

39. Torquato S (2002) Random heterogeneous materials: microstructure and macroscopic properties. Springer-Verlag, New York

40. Jiao Y, Stillinger FH, Torquato S (2007) Modeling heterogeneous materials via two-point correlation functions: basic principles. Phys Rev E 76:031110

41. Kachanov LM, Nijhoff M (eds) (1987) Introduction to continuum damage mechanics. Dordrecht, Boston

42. Simo JC, Ju JW (1987) Strain and stress-based continuum damage models, part I: formulation. Int J Solids Struct 23(7):821-840

43. Chaboche JL (1981) Continuum damage mechanics: a tool to describe phenomena before crack initiation. Nucl Engrg Des 64:233-247

44. Kevin AB, Richard B, Nicholas AW (2010) The static and high strain rate behaviour of a commingled e-glass/polypropylene woven fabric composite. Comp Sci Tech 70(2):272-283

45. Lemaitre J, Chaboche JL (1990) Mechanics of solids. Cambridge University Press, Cambridge, United Kingdom

46. Zdenek PB, Gilles Pijaudier C (1988) Nonlocal continuum damage, localization instability and convergence. J Appl Mech 55(2):287-293

47. Swaminathan S, Pagano NJ, Ghosh S (2006) Analysis of interfacial debonding in three-dimensional composite microstructures. J Engng Mater Tech 128:96-106

48. Camacho GT, Ortiz M (1996) Computational modeling of impact damage in brittle materials. Int J Solids Struct 33:2899-2938

49. Ortiz M, Pandolfi A (1999) Finite-deformation irreversible cohesive element for three-dimensional crack-propagation analysis. Int J Numer Meth Engng 44:1267-1282

50. Chow CL, Wang J (1987) An anisotropic theory of elasticity for continuum damage mechanics. Int J Frac 20:381-390

51. Cordebois JP, Sidoroff F (1982) Endommagement anisotrope et plasticite. Journal de Mechanique Theorique et Appliquee, Numero Special:45-60

52. Voyiadjis GZ, Kattan PI (2006) Advances in damage mechanics: metals and metal matrix composites with an introduction to fabric tensors. 2nd edn. Elsevier, Oxford

53. Voyiadjis GZ, Kattan PI (1992) A plasticity-damage theory for large deformation of solids. Part l: theoretical formulation. Int J Engrg Sci 30(9):1089-1108

54. Murakami S (1988) Mechanical modeling of material damage. J Appl Mech 55:280-286

55. Voyiadjis GZ, Kattan PI (1996) On the symmetrization of the effective stress tensor in continuum damage mechanics. J Mech Behav Mater 7(2):139-165

56. Rizzi E, Carol I, Willam K (1994) A unified theory of elastic degradation and damage based on a loading surface. Int J Sol Struct 31(20):2835-2865

57. Nam MD, Thanh TC (2003) Approximation of function and its derivatives using radial basis function networks. Appl Math Model 27(3):197-220 